Pacific Journal of Mathematics

ESSENTIAL SPECTRUM $\Gamma(\beta)$ OF A DUAL ACTION ON A YON 


\title{
ESSENTIAL SPECTRUM $\Gamma(\beta)$ OF A DUAL ACTION ON A VON NEUMANN ALGEBRA
}

\author{
YOSHIOMI NAKAGAMI
}

For a dual action $\beta$ of a locally compact group $G$ on a von Neumann algebra $N$ we define the essential spectrum $\Gamma(\beta)$ as the intersection of all spectrum $\mathrm{sp} \beta^{p}$ of the restriction $\beta^{p}$ of $\beta$ to $N_{p}$ when $p$ runs over all nonzero projections in $N^{\beta} . \Gamma(\beta)$ is then an algebraic invariant for a covariant dual system $\{N, \beta\} . \Gamma(\beta)$ is a closed subgroup of $G$ (Theorem 3.7). We introduce three kinds of concept for $\beta$ such as integrable, regular and dominant $(\$ \S 4,5)$. The former concepts are weaker than the dominance. If $\beta$ is regular, $\Gamma(\beta)$ coincides with the kernel of the action $\hat{\beta}$ on the center of the crossed dual product $N \otimes_{\beta}^{d} G$ (Theorem 6.1). If $\beta$ is regular, $\Gamma(\beta)$ is normal and $\Gamma(\beta)=$ $\Gamma(\tilde{\beta})$. If $\beta$ is ergodic on the center $Z(N)$ and $\Gamma(\tilde{\beta})=G$, then $N \otimes_{\beta}^{d} G$ is a factor and vice versa (Theorem 6.4). If $\beta$ is regular, $\Gamma(\beta)=G$ is equivalent to $Z\left(N^{\beta}\right) \subset Z(N)$ (Proposition 6.3). If $\beta$ is integrable on a factor $N$ and if $\Gamma(\beta)=G$, then there is a lattice isomorphism between the closed subgroups of $G$ and the von Neumann subalgebras of $N$ containing $N^{\beta}$ (Theorem 8.4). Moreover, by $N \bigotimes_{\beta}^{d}(H \backslash G)$ we mean the von Neumann algebra generated by $\beta(N)$ and $1 \otimes\left(L^{\infty}(G) \cap \lambda^{\prime}(H)^{\prime}\right)$, where $H$ is a closed subgroup of $G$ and $\lambda^{\prime}$ is the right regular representation of $G . \quad N \otimes_{\beta}^{d}(H \backslash G)$ coincides with the set of $x \in N \bigotimes_{\beta}^{d} G$ such that $\hat{\beta}_{t}(x)=x$ for all $t \in H$ (Theorem 7.2).

0. Introduction. In our previous paper $[\mathbf{1 7}, \mathbf{1 6}, \mathbf{2 1}]$ we have generalized Takesaki's duality to a general locally compact group in terms of a dual action and a crossed dual product as the following:

$$
\begin{aligned}
& \left(M \otimes_{\alpha} G\right) \otimes_{\hat{\alpha}}^{d} G \sim M \otimes B\left(L^{2}(G)\right) \\
& \left(N \otimes_{\beta}^{d} G\right) \otimes_{\hat{\beta}} G \sim N \otimes B\left(L^{2}(G)\right) .
\end{aligned}
$$

In this paper we continue our study on dual actions and Takesaki's duality obtained in the above from the view point of covariant systems $\{M, \alpha\}$ and covariant dual systems $\{N, \beta\}$. Then we naturally raise some questions:

a. What is an invariant of equivalent covariant dual systems?

b. When does Takesaki's duality hold as a covariant (dual) system? Using the spectrum of $\beta$ given in $[17, \S 5]$, we can define the essential spectrum $\Gamma(\beta)$ of $\beta$ in $\$ 3$ by the same manner as $S$ set. Then $\Gamma(\beta)$ is a closed subgroup of $G$ and an algebraic invariant of dual actions on a 
given von Neumann algebra. Therefore we can classify the dual actions into the family of closed subgroups of $G$.

c. In case of $\Gamma(\beta)=G$, what can we say about $\beta, N^{\beta}$ and $N \bigotimes_{\beta}^{d} G$ ?

For these questions Connes and Takesaki have obtained several interesting results in their paper [6] from the stand point of nonabelian cohomology in properly infinite von Neumann algebras. Following these ideas, we try to reproduce their Chapter III in terms of covariant (dual) systems. Our contents consist of following nine sections:
0 . Introduction
1. Preliminary
2. Takesaki's duality
3. Essential spectrum $\Gamma(\beta)$
4. Integrable actions and dual actions
5. Regular actions and dual actions
6. Ergodic actions and dual actions
7. Subgroups and subalgebras
8. Galois correspondence
9. Appendix

More precisely, in $\S 4$ we shall recall two equivalent conditions given by Connes and Takesaki for an action to be integrable. The dual version of one of the conditions is utilized to define the integrability of a dual action. However, we are still unclear, whether the both dual versions of the above two conditions are equivalent or not.

In $\S 5$, for the sake of convenience, we call an action or a dual action to be regular when it is dual to some dual action or some action. The regularity is then stronger than integrability. Their characterizations are already given by Landstad and others, $[\mathbf{1 5}, \mathbf{1 7}, \mathbf{1 6}, \mathbf{2 1}]$. Furthermore, we call a (dual) action to be dominant if it is regular and of infinite multiplicity. The dominance in our paper is a generalization of the one given by Connes and Takesaki to a non separable case. The utility of regularity and dominance will become clearer in our later sections as we analyze the equivalence class of covariant (dual) systems.

In $\S 6, \Gamma(\hat{\alpha})$ turns out to be the set of all $t \in G$ such that $\alpha_{t}$ is trivial on the center of $M$. Therefore, if $\beta \equiv \hat{\alpha}, \Gamma(\beta)=H$ is characterized by the fact that $H$ is the largest closed subgroup satisfying that the center of $N \bigotimes_{\beta}^{d} G$ commutes with $1 \otimes \lambda^{\prime}(H)$, where $\lambda^{\prime}$ is the left regular representation of $G$. Further, if $\beta$ is ergodic on the center of $N$, then $N^{\beta}$ is a factor, in addition, if $\Gamma(\tilde{\beta})=G$, then $N \otimes_{\beta}^{d} G$ is a factor.

Let $M \otimes_{\alpha} H$ be the von Neumann algebra generated by $\alpha(M)$ and $1 \otimes \lambda(H)$, and let $N \bigotimes_{\beta}^{d}(H \backslash G)$ be the von Neumann algebra generated by $\beta(N)$ and $\mathscr{L}^{\infty}(H \backslash G)$, where $\mathscr{L}^{\infty}(H \backslash G) \equiv L^{\infty}(G) \cap \lambda^{\prime}(H)^{\prime}$. In $\S 7$ we shall characterize these von Neumann subalgebras, namely, $M \otimes_{\alpha} H$ is the set of $y$ in $M \otimes_{\alpha} G$ with $\hat{\alpha}(y) \subset N \otimes \lambda(H)^{\prime \prime}$ and $N \bigotimes_{\beta}^{d}(H \backslash G)$ is the set of $x$ in $N \bigotimes_{\beta}^{d} G$ with $\hat{\beta}_{t}(x)=x$ for all $t \in H$. 
In $\$ 8$ we shall give a Galois type correspondence between the closed subgroups of $G$ and the von Neumann subalgebras of a factor $N$ including $N^{\beta}$ under the assumption that $G$ is separable, $\beta$ is integrable and $\Gamma(\beta)=G$. Let $\beta^{d}$ be a dual action of $G$ on $N \bigotimes_{\beta}^{d} G$ given by (1.8). If $N \bigotimes_{\beta}^{d} G$ is a factor and if $L$ is a $\beta^{d}$ invariant von Neumann subalgebra of $N \bigotimes_{\beta}^{d} G$ including $\beta(N)$, then $L$ is of the form $N \otimes_{\beta}^{d}(H \backslash G)$ for some closed subgroup $H$ of $G$. Conversely, $N \otimes_{\beta}^{d}(H \backslash G)$ is $\beta^{d}$ invariant.

In the Appendix we shall give a sufficient condition for an action of a locally compact abelian group to be regular.

The author wants to express his sincere gratitude to Professors $\mathrm{M}$. Takesaki and Y. Oka for their valuable discussion and to Professor M. Tomita for his encouragement.

1. Preliminary. Let $G$ be a locally compact group, $d t$ the Haar measure, $t \mapsto \lambda(t)$ the right regular representation of $G$ on $L^{2}(G)$ and $R(G)$ the von Neumann algebra generated by $\lambda(G)$. Let $J$ be the antiunitary involution on $L^{2}(G)$ defined by $\left(J^{c} \xi\right)(t) \equiv \Delta(t)^{1 / 2} \overline{\xi\left(t^{-1}\right)}$ for $\xi \in L^{2}(G)$ and $\lambda^{\prime}(r) \equiv J^{c} \lambda(r) J^{c}$, where $\Delta$ is the modular function. Then $\lambda^{\prime}$ is the left regular representation:

$$
\left(\lambda^{\prime}(r) \xi\right)(t)=\Delta(r)^{1 / 2} \xi\left(r^{-1} t\right) .
$$

Let $M$ and $N$ be von Neumann algebras acting on Hilbert spaces $\mathscr{H}$ and $\mathscr{K}$, respectively. $\quad \gamma$ and $\delta$ are isomorphisms $R(G) \rightarrow R(G) \otimes R(G)$ and $L^{\star}(G) \rightarrow L^{\infty}(G) \otimes L^{\infty}(G)$ satisfying

$$
\gamma \lambda(r)=\lambda \quad r) \otimes \lambda(r) \quad \text { and } \quad(\delta f)(s, t)=f(s t)
$$

for $f \in L^{\star}(G)$. An acti in $\alpha$ of $G$ on $M$ is an isomorphism of $M$ into $M \otimes L^{\times}(G)$ satisfying

$$
\alpha \otimes \iota \circ \alpha=\imath \otimes \delta \circ \alpha
$$

where $\iota$ denotes the identity automorphism and the association of the tensor product $\otimes$ is stronger than that of the composition $\circ$. By [17, Theorem 2.1] an action $\alpha$ is induced from an action $t \mapsto \alpha_{t}$ of $G$ on $M$ in the usual sense by $(\alpha(x) \xi)(t)=\alpha_{t}(x) \xi(t)$ for $\xi \in \mathscr{H} \otimes L^{2}(G)$. As $\alpha\left(\alpha_{t}(x)\right)=A d 1 \otimes \lambda(t)(\alpha(x))$ for all $t,\{M, \alpha\}$ is a covariant system. A dual action $\beta$ of $G$ on $N$ is defined as an isomorphism of $N$ into $N \otimes R(G)$ satisfying

$$
\beta \otimes \iota \circ \beta=\imath \otimes \gamma \circ \beta
$$

Such a pair $\{N, \beta\}$ is called a covariant dual system. 
The crossed product $M \otimes_{\alpha} G$ of $M$ by $G$ with respect to $\alpha$ is the von Neumann algebra generated by $\alpha(M)$ and $1 \otimes R(G)$. The crossed dual product $N \otimes_{\beta}^{d} G$ of $N$ and $G$ with respect to $\beta$ is defined as the von Neumann algebra generated by $\beta(N)$ and $1 \otimes L^{\infty}(G)$.

Now we define a unitary $W$ on $L^{2}(G) \otimes L^{2}(G)$ by

$$
(W \xi)(s, t) \equiv \xi(s, t s)
$$

Then $W^{*}=A d J \otimes 1(W)$, where $(J \xi)(t) \equiv \Delta(t)^{1 / 2} \xi\left(t^{-1}\right)$ for $\xi \in L^{2}(G)$. We set

$$
W^{\prime} \equiv A d 1 \otimes J(W), \quad V \equiv \sigma(W) \quad \text { and } \quad V^{\prime} \equiv \sigma\left(W^{\prime}\right),
$$

where $\sigma$ denotes the symmetric isomorphism

$$
x \otimes y \mapsto y \otimes x
$$

These unitaries satisfy $\left(W^{\prime} \xi\right)(s, t)=\Delta(s)^{1 / 2} \xi\left(s, s^{-1} t\right),(V \xi)(s, t)=\xi(s t, t)$ and $\left(V^{\prime} \xi\right)(s, t)=\Delta(t)^{1 / 2} \xi\left(t^{-1} s, t\right)$. Therefore (1.1) and (1.2) are of the forms

$$
\begin{aligned}
& \alpha \otimes \iota \circ \alpha(x)=A d 1 \otimes V\left(\alpha(x) \otimes 1_{G}\right) \\
& \beta \otimes \iota \circ \beta(y)=A d 1 \otimes W^{*}\left(\beta(y) \otimes 1_{G}\right) .
\end{aligned}
$$

On the other hand, since

$$
\begin{aligned}
& \text { Ad } 1 \otimes W^{*}\left(\alpha(x) \otimes 1_{G}\right)=\alpha(x) \otimes 1_{G}, \\
& \text { Ad } 1 \otimes W^{*}\left(\lambda_{1}(r) \otimes 1_{G}\right)=\lambda_{1}(r) \otimes \lambda(r) \\
& \text { Ad } 1 \otimes V^{\prime}\left(\beta(y) \otimes 1_{G}\right)=\beta(y) \otimes 1_{G}, \\
& \text { Ad } 1 \otimes V^{\prime}\left(1 \otimes f \otimes 1_{G}\right)=1 \otimes \epsilon f,
\end{aligned}
$$

where $\lambda_{1}(r) \equiv 1 \otimes \lambda(r)$ and $(\epsilon f)(s, t) \equiv f\left(t^{-1} s\right)$, it follows that

$$
\begin{aligned}
& \text { Ad } 1 \otimes W^{*}\left(\left(M \otimes_{\alpha} G\right) \otimes 1_{G}\right) \subset\left(M \otimes_{\alpha} G\right) \otimes R(G) \\
& \text { Ad } 1 \otimes V^{\prime}\left(\left(N \otimes_{\beta}^{d} G\right) \otimes 1_{G}\right) \subset\left(N \otimes_{\beta}^{d} G\right) \otimes L^{\circ}(G) .
\end{aligned}
$$

We denote their restrictions by $\hat{\alpha}$ and $\hat{\beta}$ :

$$
\begin{aligned}
& \hat{\alpha}(x) \equiv A d 1 \otimes W^{*}\left(x \otimes 1_{G}\right) \\
& \hat{\beta}(y) \equiv A d 1 \otimes V^{\prime}\left(y \otimes 1_{G}\right)
\end{aligned}
$$

for $x \in M \otimes_{\alpha} G$ and $y \in N \bigotimes_{\beta}^{d} G$. Since 


$$
\begin{aligned}
& W^{*} \otimes 1_{G}(\iota \otimes \sigma)\left(W^{*} \otimes 1_{G}\right)=A d 1_{G} \otimes W^{*}\left(W^{*} \otimes 1_{G}\right) \\
& V^{\prime} \otimes 1_{G}(\iota \otimes \sigma)\left(V^{\prime} \otimes 1_{G}\right)=A d 1_{G} \otimes V\left(V^{\prime} \otimes 1_{G}\right),
\end{aligned}
$$

it is shown in [17, Theorem 2.3] that $\hat{\alpha}$ is a dual action of $G$ on $M \otimes_{\alpha} G$ and $\hat{\beta}$ is an action of $G$ on $N \otimes_{\beta}^{d} G$. $\quad \hat{\alpha}$ and $\hat{\beta}$ are said to be the dual action dual to $\alpha$ and the action dual to $\beta$. We denote the action dual to $\hat{\alpha}$ by $\hat{\hat{\alpha}}$ or $\tilde{\alpha}$ and the dual action dual to $\hat{\beta}$ by $\hat{\beta}$ or $\tilde{\beta}$.

We know from (1.3) that

$$
\begin{aligned}
& A d 1 \otimes V\left(\alpha(M) \otimes 1_{G}\right) \subset \alpha \otimes \iota\left(M \otimes L^{\infty}(G)\right) \\
& A d 1 \otimes W^{*}\left(\beta(N) \otimes 1_{G}\right) \subset \beta \otimes \iota(N \otimes R(G)) .
\end{aligned}
$$

Since $\left[V, \lambda^{\prime}(r) \otimes f\right]=0$ and $\left[W, f \otimes 1_{G}\right]=0$, it follows that

$$
\begin{aligned}
& A d 1 \otimes V\left(1 \otimes R(G) \otimes 1_{G}\right) \subset 1 \otimes R(G) \otimes L^{\infty}(G) \\
& A d 1 \otimes W^{*}\left(1 \otimes L^{\infty}(G) \otimes 1_{G}\right)=1 \otimes L^{\infty}(G) \otimes 1_{G} .
\end{aligned}
$$

Therefore

$$
\begin{aligned}
& A d 1 \otimes V\left(\left(M \otimes_{\alpha} G\right) \otimes 1_{G}\right) \subset\left(M \otimes_{\alpha} G\right) \otimes L^{\infty}(G) \\
& A d 1 \otimes W^{*}\left(\left(N \otimes_{\beta}^{d} G\right) \otimes 1_{G}\right) \subset\left(N \otimes_{\beta}^{d} G\right) \otimes R(G),
\end{aligned}
$$

which is compared with (1.4). If we define $\alpha^{d}$ and $\beta^{d}$ by

$$
\begin{aligned}
& \alpha^{d}(x) \equiv A d 1 \otimes V\left(x \otimes 1_{G}\right) \\
& \beta^{d}(y) \equiv A d 1 \otimes W^{*}\left(y \otimes 1_{G}\right)
\end{aligned}
$$

for $x \in M \otimes_{\alpha} G$ and $y \in N \otimes_{\beta}^{d} G$, then $\alpha^{d}$ is an action of $G$ on $M \otimes_{\alpha} G$ and $\beta^{d}$ is a dual action of $G$ on $N \bigotimes_{\beta}^{d} G$. Indeed, (1.3) for $\alpha^{d}$ and $\beta^{d}$ is proved by

$$
V \otimes 1_{G}(\iota \otimes \sigma)\left(V \otimes 1_{G}\right)=A d 1_{G} \otimes V\left(V \otimes 1_{G}\right)
$$

and (1.6) for $W$.

On the other hand, since $[V, 1 \otimes f]=0$ and $\left[W, 1 \otimes \lambda^{\prime}(r)\right]=0$, it follows from (1.7) that

$$
\begin{aligned}
& \text { Ad } 1 \otimes V^{*}\left(\alpha(M)^{\prime} \otimes 1_{G}\right) \subset \alpha(M)^{\prime} \otimes L^{\infty}(G) \\
& \text { Ad } 1 \otimes W\left(\beta(N)^{\prime} \otimes 1_{G}\right) \subset \beta(N)^{\prime} \otimes R(G) .
\end{aligned}
$$

If we define $\alpha^{\prime}$ and $\beta^{\prime}$ by

$$
\begin{aligned}
& \alpha^{\prime}(x) \equiv A d 1 \otimes V^{*}\left(x \otimes 1_{G}\right) \\
& \beta^{\prime}(y) \equiv A d 1 \otimes W\left(y \otimes 1_{G}\right)
\end{aligned}
$$


for $x \in \alpha(M)^{\prime}$ and $y \in \beta(N)^{\prime}$, then we have the following theorem due to Landstad, [16, the proof of Lemma 1].

THEOREM 1.1. $\quad \beta^{\prime}$ is a dual action of $G$ on $\beta(N)^{\prime}$ satisfying

(i) $\left(\beta(N)^{\prime}\right)^{\beta^{\prime}}=\left(N \bigotimes_{\beta}^{d} G\right)^{\prime}$; and

(ii) there is an action $\alpha$ of $G$ on $\left(N \bigotimes_{\beta}^{d} G\right)^{\prime}$ satisfying

$$
\left\{\beta(N)^{\prime}, \beta^{\prime}\right\} \sim\left\{\left(N \otimes_{\beta}^{d} G\right)^{\prime} \otimes_{\alpha} G, \hat{\alpha}\right\}
$$

where $\left(\beta(N)^{\prime}\right)^{\beta^{\prime}} \equiv\left\{y \in \beta(N)^{\prime}: \beta^{\prime}(y)=y \otimes 1_{G}\right\}$.

As this theorem is important from the technical point of view, we shall give a dual version, although it is unnecessary for our later use.

An isomorphism $\alpha$ of $M$ into $M \otimes L^{\infty}(G)$ satisfying $\alpha \otimes \iota \circ \alpha=$ $\iota \otimes \delta^{c} \circ \alpha\left(\delta^{c} f \equiv A d V^{*}\left(f \otimes 1_{G}\right)\right)$ is called an action of $G$ on $M$ with respect to $R(G)^{\prime},[\mathbf{1 7}$, Appendix].

THEOREM 1.2. $\alpha^{\prime}$ is an action of $G$ on $\alpha(M)^{\prime}$ with respect to $R(G)^{\prime}$ satisfying

(i) $\left(\alpha(M)^{\prime}\right)^{\alpha^{\prime}}=\left(M \otimes_{\alpha} G\right)^{\prime} ;$ and

(ii) there is a dual action $\beta^{c}$ of $G$ on $\left(M \otimes_{\alpha} G\right)^{\prime}$ with respect to $R(G)^{\prime}$ such that

$$
\left\{\alpha(M)^{\prime}, \alpha^{\prime}\right\} \sim\left\{\left(M \otimes_{\alpha} G\right\}^{\prime} \bigotimes_{\beta^{c}}^{d} G, \hat{\beta}^{c}\right\}
$$

Proof. Since

$$
\left(V^{*} \otimes 1_{G}\right) \iota \otimes \sigma\left(V^{*} \otimes 1_{G}\right)=A d 1_{G} \otimes V^{*}\left(V^{*} \otimes 1_{G}\right),
$$

we have

$$
\begin{aligned}
& \alpha^{\prime} \otimes \iota \circ \alpha^{\prime}(x) \\
& \quad=A d 1 \otimes V^{*} \otimes 1_{G} \circ \iota \otimes \iota \otimes \sigma \circ A d 1 \otimes V^{*} \otimes 1_{G}\left(x \otimes 1_{G} \otimes 1_{G}\right) \\
& \quad=A d 1 \otimes 1_{G} \otimes V^{\prime *} \circ A d 1 \otimes V^{*} \otimes 1_{G}\left(x \otimes 1_{G} \otimes 1_{G}\right) \\
& \quad=\iota \otimes \delta^{c} \circ \alpha^{\prime}(x),
\end{aligned}
$$

where $\delta^{c}(f) \equiv A d V^{* *}\left(f \otimes 1_{G}\right)$ for $f \in L^{\infty}(G)$. Therefore $\alpha^{\prime}$ is an action of $G$ on $\alpha(M)^{\prime}$ with respect to $R(G)^{\prime}$.

(i) Put $N \equiv\left(\alpha(M)^{\prime}\right)^{\alpha^{\prime}}$. Since $M$ is standard, we have a weakly continuous unitary representation $u$ of $G$ on $\mathscr{H}$ implementing $\alpha$, [1,9]. $\left(M \otimes_{\alpha} G\right)^{\prime}$ is then generated by $M^{\prime} \otimes 1_{G}$ and $U\left(1 \otimes R(G)^{\prime}\right) U^{*}$, where $U$ is a unitary on $\mathscr{H} \otimes L^{2}(G)$ defined by $(U \xi)(s) \equiv u(s) \xi(s)$, $[7,10,16]$. As $\left(M \otimes_{\alpha} G\right)^{\prime} \subset N$ is clear, it suffices to show the converse inclusion. For this we suppose that $x \in N$. Then $x \in \alpha(M)^{\prime}$ and 
Ad $1 \otimes V^{*}\left(x \otimes 1_{G}\right)=x \otimes 1_{G} . \quad$ Therefore

$$
\begin{aligned}
x \otimes 1_{G} & \in\left(1 \otimes 1_{G} \otimes R(G)\right)^{\prime} \cap A d 1 \otimes V^{*}\left(1 \otimes 1_{G} \otimes R(G)\right)^{\prime} \\
& =(1 \otimes R(G) \otimes R(G))^{\prime}
\end{aligned}
$$

and hence

$$
x \in \alpha(M)^{\prime} \cap(1 \otimes R(G))^{\prime}=\left(M \otimes_{\alpha} G\right)^{\prime} .
$$

(ii) Since $\alpha(M)=\left(M \otimes_{\alpha} G\right)^{\hat{\alpha}}$ by (2.7) and since $\alpha(M)=$ $\left(M \otimes_{\alpha} G\right) \cap\left(1 \otimes L^{\infty}(G)\right)^{\prime}$ by Theorem 7.1 , it follows that

$$
\alpha(M)^{\prime}=\left(\left(M \otimes_{\alpha} G\right)^{\prime} \cup\left(1 \otimes L^{\infty}(G)\right)\right)^{\prime \prime} .
$$

Moreover,

$$
\alpha_{t}^{\prime}(1 \otimes f)=A d 1 \otimes \lambda(t)^{*}(1 \otimes f)
$$

for $f \in L^{\infty}(G) . \quad$ By $[16: 17$, Theorem $8.3 ; 18]$ we have the desired result.

Finally, we recall the equivalence used in the above theorems. Let $\alpha^{\prime}$ (resp. $\beta_{\jmath}$ ) be an action (resp. a dual action) of $G$ on $M_{J}$ (resp. $N_{\jmath}$ ) for $j=1,2$. We denote

$$
\left\{M_{1}, \alpha^{1}\right\} \sim\left\{M_{2}, \alpha^{2}\right\} \quad\left(\text { resp. }\left\{N_{1}, \beta_{1}\right\} \sim\left\{N_{2}, \beta_{2}\right\}\right),
$$

if there exists an isomorphism $\rho$ of $M_{1}\left(\operatorname{resp} . N_{1}\right)$ onto $M_{2}\left(\operatorname{resp} . N_{2}\right)$ satisfying

$$
\rho \otimes \iota \circ \alpha^{1}=\alpha^{2} \circ \rho \quad\left(\text { resp. } \rho \otimes \iota \circ \beta_{1}=\beta_{2} \circ \rho\right)
$$

Then it is direct from (1.1) and (1.2) that

$$
\{M, \alpha\} \sim\{\alpha(M), \iota \otimes \delta\} \quad \text { and } \quad\{N, \beta\} \sim\{\beta(N), \iota \otimes \gamma\}
$$

2. Takesaki's duality. In this section we shall give a few supplements to our previous results obtained in [17].

We begin by recalling Takesaki's duality $[16, \mathbf{1 7}, \mathbf{1 8}]$. If $\pi_{M}$ is an isomorphism of $M$ into $\left(M \bigotimes_{\alpha} G\right) \bigotimes_{\dot{\alpha}}^{d} G$ given by

$$
\pi_{M}(x) \equiv A d 1 \otimes V^{*}\left(\alpha(x) \otimes 1_{G}\right)
$$

for $x$ in $M$, then $\left(M \otimes_{\alpha} G\right) \bigotimes_{\dot{\alpha}}^{d} G$ is generated by $\pi_{M}(x)(x \in M)$, $1 \otimes \lambda(r) \otimes \lambda(r)(r \in G)$ and $1 \otimes 1_{G} \otimes f\left(f \in L^{\infty}(G)\right),[17]$. We denote 
by $\rho$ an isomorphism of $M \otimes B\left(L^{2}(G)\right)$ onto $\left(M \bigotimes_{\alpha} G\right) \bigotimes_{\hat{\alpha}}^{d} G$ defined by Ad $1 \otimes V^{* \circ \alpha} \otimes \iota$

Then $\rho\left(x \otimes 1_{G}\right)=\pi_{M}(x), \rho(1 \otimes \lambda(r))=1 \otimes \lambda(r) \otimes \lambda(r)$ and $\rho(1 \otimes f)=$ $1 \otimes 1_{G} \otimes f$.

THEOREM 2.1. If $\alpha$ is an action of $G$ on $M$, then

$$
\left\{\left(M \otimes_{\alpha} G\right) \bigotimes_{\tilde{\alpha}}^{d} G, \tilde{\alpha}\right\} \sim\left\{M \otimes B\left(L^{2}(G)\right),[\alpha]\right\},
$$

where $\quad[\alpha] \equiv$ Ad $1 \otimes V^{\prime} \circ \iota \otimes \sigma \circ \alpha \otimes \iota$. In particular, $\quad[\alpha]_{t}=$ $\alpha_{t} \otimes A d \lambda^{\prime}(t)$ for $t \in G$.

LEMMA 2.2. If $\rho$ is given by (2.1), then

$$
\tilde{\alpha} \circ \rho=\rho \otimes \iota \circ A d 1 \otimes V^{\prime} \circ \bar{\alpha}
$$

on $M \otimes 1_{G}$, where $\bar{\alpha} \equiv \iota \otimes \sigma \circ \alpha \otimes \iota$.

Proof. Since

$$
V^{*} \otimes 1_{G}(\iota \otimes \sigma)\left(V^{*} \otimes 1_{G}\right)=A d 1_{G} \otimes V^{*}\left(V^{*} \otimes 1_{G}\right),
$$

we have

(2.3) $\left(1_{G} \otimes V^{\prime}\right)\left(V^{*} \otimes 1_{G}\right)=\left(V^{*} \otimes 1_{G}\right)\left(1_{G} \otimes V^{\prime}\right) \iota \otimes \sigma\left(V \otimes 1_{G}\right)$.

If $x \in M$, then $\iota \otimes \sigma\left(x \otimes 1_{G} \otimes 1_{G}\right)=x \otimes 1_{G} \otimes 1_{G}$. Therefore

$$
\begin{aligned}
& \rho \otimes \iota \circ A d 1 \otimes V^{\prime} \circ \bar{\alpha}\left(x \otimes 1_{G}\right) \\
&= A d 1 \otimes V^{*} \otimes 1_{G} \circ \alpha \otimes \iota \otimes \iota \circ A d 1 \otimes V^{\prime} \circ \bar{\alpha}\left(x \otimes 1_{G}\right) \quad(\text { By }(2.1)) \\
&= A d\left(\left(1 \otimes V^{*} \otimes 1_{G}\right)\left(1 \otimes 1_{G} \otimes V^{\prime}\right)\right) \circ \otimes \otimes \iota \otimes \iota \circ \bar{\alpha}\left(x \otimes 1_{G}\right) \\
&= A d\left(\left(1 \otimes V^{*} \otimes 1_{G}\right)\left(1 \otimes 1_{G} \otimes V^{\prime}\right) \iota \otimes \iota \otimes \sigma\left(1 \otimes V \otimes 1_{G}\right)\right) \\
& \circ \alpha \otimes \iota \otimes \iota\left(x \otimes 1_{G} \otimes 1_{G}\right) \quad(\mathrm{By}(1.3)) \\
&= \operatorname{Ad}\left(\left(1 \otimes 1_{G} \otimes V^{\prime}\right)\left(1 \otimes V^{*} \otimes 1_{G}\right)\right) \circ \alpha \otimes \iota \otimes \iota\left(x \otimes 1_{G} \otimes 1_{G}\right)
\end{aligned}
$$

$$
\begin{aligned}
& =A d 1 \otimes 1_{G} \otimes V^{\prime} \circ \rho \otimes \iota\left(x \otimes 1_{G} \otimes 1_{G}\right) \quad(\text { By (2.1)) } \\
& =\tilde{\alpha} \circ \rho\left(x \otimes 1_{G}\right) .
\end{aligned}
$$

This completes the proof. 
Proof of Theorem 2.1. It is immediate from the following calculation:

$$
\begin{aligned}
(\rho \otimes \iota)^{-1} \circ \tilde{\alpha} \circ \rho(1 \otimes \lambda(r)) & =(\rho \otimes \iota)^{-1} \circ \tilde{\alpha}(1 \otimes \lambda(r) \otimes \lambda(r)) \\
& =(\rho \otimes \iota)^{-1}\left(1 \otimes \lambda(r) \otimes \lambda(r) \otimes 1_{G}\right) \\
& =1 \otimes \lambda(r) \otimes 1_{G}=[\alpha](1 \otimes \lambda(r))
\end{aligned}
$$

and

$$
\begin{aligned}
(\rho \otimes \iota)^{-1} \circ \tilde{\alpha} \circ \rho(1 \otimes f) & =(\rho \otimes \iota)^{-1} \circ \tilde{\alpha}\left(1 \otimes 1_{G} \otimes f\right) \\
& =(\rho \otimes \iota)^{-1}\left(1 \otimes 1_{G} \otimes \epsilon f\right) \\
& =1 \otimes \epsilon f=[\alpha](1 \otimes f) .
\end{aligned}
$$

This completes the proof.

The dual version of the above result is obtained as follows. If $\pi_{N}$ is an isomorphism of $N$ into $\left(N \bigotimes_{\beta}^{d} G\right) \otimes_{\beta} G$ given by

$$
\pi_{N}(y) \equiv A d 1 \otimes W^{\prime}\left(\beta(y) \otimes 1_{G}\right)
$$

for $y$ in $N$, then $\left(N \otimes_{\beta}^{d} G\right) \bigotimes_{\hat{\beta}} G$ is generated by $\pi_{N}(y)(y \in N), 1 \otimes \epsilon f$ $\left(f \in L^{\infty}(G)\right)$ and $1 \otimes 1_{G} \otimes \lambda(r)(r \in G),[17]$. We denote by $\rho$ an isomorphism of $N \otimes B\left(L^{2}(G)\right)$ onto $\left(N \bigotimes_{\beta}^{d} G\right) \bigotimes_{\beta} G$ defined by

$$
\text { Ad } 1 \otimes 1_{G} \otimes J \circ A d 1 \otimes W \circ \beta \otimes \iota \text {. }
$$

where $J$ is given by $(J \xi)(t) \equiv \Delta(t)^{1 / 2} \xi\left(t^{-1}\right)$ for $\xi \in L^{2}(G)$. Then $\rho\left(y \otimes 1_{G}\right)=\pi_{N}(y), \rho(1 \otimes f)=1 \otimes \epsilon f$ and $\rho\left(1 \otimes \lambda^{\prime}(r)\right)=1 \otimes 1_{G} \otimes \lambda(r)$.

THEOREM 2.3. If $\beta$ is a dual action on $N$, then

$$
\left\{\left(N \otimes_{\beta}^{d} G\right) \bigotimes_{\hat{\beta}} G, \tilde{\beta}\right\} \sim\left\{N \otimes B\left(L^{2}(G)\right),[\beta]\right\},
$$

where $[\beta] \equiv A d 1 \otimes W \circ \iota \otimes \sigma \circ \beta \otimes \iota$.

Lemma 2.4. If $\rho$ is defined by (2.4), then

$$
\tilde{\beta} \circ \rho=\rho \otimes \iota \circ A d 1 \otimes W \circ \bar{\beta}
$$

on $N \otimes 1_{G}$, where $\bar{\beta} \equiv \iota \otimes \sigma \circ \beta \otimes \iota$.

Proof. Since

$$
W \otimes 1_{G}(\iota \otimes \sigma)\left(W \otimes 1_{G}\right)=A d 1_{G} \otimes W^{*}\left(W \otimes 1_{G}\right),
$$


we have

$\left(W \otimes 1_{G}\right)\left(1_{G} \otimes W\right) \iota \otimes \sigma\left(W^{*} \otimes 1_{G}\right)=\left(1_{G} \otimes W\right)\left(W \otimes 1_{G}\right)$.

If $y \in N$, then $\iota \otimes \sigma\left(y \otimes 1_{G} \otimes 1_{G}\right)=y \otimes 1_{G} \otimes 1_{G}$. Therefore $A d 1 \otimes 1_{G} \otimes J \otimes 1_{G} \circ \rho \otimes \iota \circ A d 1 \otimes W \circ \bar{\beta}\left(y \otimes 1_{G}\right)$

$=A d 1 \otimes W \otimes 1_{G} \circ \beta \otimes \iota \otimes \iota \circ A d 1 \otimes W \circ \bar{\beta}\left(y \otimes 1_{G}\right) \quad$ (By (2.4))

$=A d\left(\left(1 \otimes W \otimes 1_{G}\right)\left(1 \otimes 1_{G} \otimes W\right)\right) \circ \beta \otimes \iota \otimes \iota \circ \bar{\beta}\left(y \otimes 1_{G}\right)$

$=A d\left(\left(1 \otimes W \otimes 1_{G}\right)\left(1 \otimes 1_{G} \otimes W\right)\right)$

$\circ \iota \otimes \iota \otimes \sigma \circ \beta \otimes \iota \otimes \iota \circ \beta \otimes \iota\left(y \otimes 1_{G}\right)$

$=A d\left(\left(1 \otimes W \otimes 1_{G}\right)\left(1 \otimes 1_{G} \otimes W\right) \iota \otimes \iota \otimes \sigma\left(1 \otimes W^{*} \otimes 1_{G}\right)\right)$

- $\beta \otimes \imath \otimes \iota\left(y \otimes 1_{G} \otimes 1_{G}\right) \quad$ (By (1.3))

$=A d\left(\left(1 \otimes 1_{G} \otimes W\right)\left(1 \otimes W \otimes 1_{G}\right)\right) \circ \beta \otimes \iota \otimes \iota\left(y \otimes 1_{G} \otimes 1_{G}\right)$

$=A d\left(\left(1 \otimes 1_{G} \otimes W\right)\left(1 \otimes 1_{G} \otimes J \otimes 1_{G}\right)\right) \circ \rho \otimes \iota\left(y \otimes 1_{G} \otimes 1_{G}\right)$

(By (2.4))

$=A d\left(\left(1 \otimes 1_{G} \otimes J \otimes 1_{G}\right)\left(1 \otimes 1_{G} \otimes W^{*}\right) \circ \rho \otimes \iota\left(y \otimes 1_{G} \otimes 1_{G}\right)\right.$

$=A d\left(1 \otimes 1_{G} \otimes J \otimes 1_{G}\right) \circ \tilde{\beta} \circ \rho\left(y \otimes 1_{G}\right)$.

This completes the proof.

Proof of Theorem 2.3. It is immediate from the

$$
\begin{aligned}
& (\rho \otimes \iota)^{-1} \circ \tilde{\beta} \circ \rho(1 \otimes f)=(\rho \otimes \iota)^{-1} \circ \tilde{\beta}(1 \otimes \epsilon f) \\
& \quad=(\rho \otimes \iota)^{-1}\left(1 \otimes \epsilon f \otimes 1_{G}\right)=1 \otimes f \otimes 1_{G}=[\beta](1 \otimes f)
\end{aligned}
$$

and

$$
\begin{aligned}
& (\rho \otimes \iota)^{-1} \circ \tilde{\beta} \circ \rho\left(1 \otimes \lambda^{\prime}(r)\right)=(\rho \otimes \iota)^{-1} \circ \tilde{\beta}\left(1 \otimes 1_{G} \otimes \lambda(r)\right) \\
& \quad=(\rho \otimes \iota)^{-1}\left(1 \otimes 1_{G} \otimes \lambda(r) \otimes \lambda(r)\right) \\
& \quad=1 \otimes \lambda^{\prime}(r) \otimes \lambda(r)=[\beta]\left(1 \otimes \lambda^{\prime}(r)\right) .
\end{aligned}
$$

This completes the proof.

By the use of operator valued weights $E_{\alpha}$ and $E_{\beta}$ we have shown in $[16 ; 17$, Proposition 6.1 and $6.4 ; 18]$ that

$$
\left(M \otimes_{\alpha} G\right)^{\hat{\alpha}}=\alpha(M) \text { and }\left(N \bigotimes_{\beta}^{d} G\right)^{\hat{\beta}}=\beta(N) .
$$


Combining (2.7) and Takesaki's duality in the above, we have the following theorem.

THEOREM 2.5. (i) If $\alpha$ is an action of $G$ on $M$, then

$$
M \otimes_{\alpha} G=\left(M \otimes B\left(L^{2}(G)\right)\right)^{[\alpha]}
$$

(ii) If $\beta$ is a dual action of $G$ on $N$, then

$$
N \otimes_{\beta}^{d} G=\left(N \otimes B\left(L^{2}(G)\right)\right)^{[\beta]} .
$$

Proof. (i) Let $\rho$ be the isomorphism of $M \otimes B\left(L^{2}(G)\right)$ onto $\left(M \otimes_{\alpha} G\right) \bigotimes_{\hat{\alpha}}^{d} G$ given by (2.1). Then, by Theorem 2.1 and (2.7), we have

$$
\rho\left(\left(M \otimes B\left(L^{2}(G)\right)\right)^{[\alpha]}\right)=\hat{\alpha}\left(M \otimes_{\alpha} G\right) .
$$

Since

$$
\rho^{-1} \circ \hat{\alpha}(\alpha(x))=(\alpha \otimes \iota)^{-1} \circ A d 1 \otimes V\left(\alpha(x) \otimes 1_{G}\right)=\alpha(x)
$$

by (1.3) and

$$
\rho^{-1} \circ \hat{\alpha}(1 \otimes \lambda(r))=\rho^{-1}(1 \otimes \lambda(r) \otimes \lambda(r))=1 \otimes \lambda(r)
$$

as before, we have (2.8).

(ii) Let $\rho$ be the isomorphism of $N \otimes B\left(L^{2}(G)\right)$ onto $\left(N \bigotimes_{\beta}^{d} G\right) \otimes_{\beta} G$ given by (2.4). Then, by Theorem 2.3 and (2.7), we have

$$
\rho\left(\left(N \otimes B\left(L^{2}(G)\right)\right)^{[\beta]}\right)=\hat{\beta}\left(N \otimes_{\beta}^{d} G\right) .
$$

Since

$$
\begin{aligned}
\rho^{-1} \circ \hat{\beta}(\beta(y)) & =(\beta \otimes \iota)^{-1} \circ \operatorname{Ad}\left(\left(1 \otimes W^{*}\right)\left(1 \otimes 1_{G} \otimes J\right)\right)\left(\beta(y) \otimes 1_{G}\right) \\
& =\beta(y)
\end{aligned}
$$

by (1.3) and

$$
\rho^{-1} \circ \hat{\beta}(1 \otimes f)=\rho^{-1}(1 \otimes \epsilon f)=1 \otimes f
$$

as before, we have (2.9).

Hereafter we shall use the following notations: 


$$
\begin{aligned}
& \bar{M} \equiv M \otimes B\left(L^{2}(G)\right), \quad \bar{\alpha} \equiv \iota \otimes \sigma \circ \alpha \otimes \iota, \quad[\alpha] \equiv A d 1 \otimes V^{\prime} \circ \bar{\alpha} \\
& \bar{N} \equiv N \otimes B\left(L^{2}(G)\right), \quad \bar{\beta} \equiv \iota \otimes \sigma \circ \beta \otimes \iota, \quad[\beta] \equiv A d 1 \otimes W \circ \bar{\beta}
\end{aligned}
$$

without any reference.

3. Essential spectrum $\Gamma(\beta)$. In this section we shall define an invariant $\Gamma(\beta)$ of a dual action $\beta$ in analogy with $\Gamma(\alpha)$ defined for an action $\alpha$ by Connes, [5]. The argument will proceed similarly as that for $\alpha$.

For a dual action $\beta$ of $G$ on $N$ we define $\beta_{\phi}$ and $\Phi_{\omega}$ for $\phi \in R(G)_{*}$ and $\omega \in N_{*}$ by

$$
\left\langle\beta_{\phi}(y), \omega\right\rangle=\langle\beta(y), \omega \otimes \phi\rangle=\left\langle\Phi_{\omega}(y), \phi\right\rangle .
$$

Let $m_{y}$ and $m$ denote the set of all $\phi \in R(G)_{*}$ with $\beta_{\phi}(y)=0$ and $\beta_{\phi}=0$, respectively. The spectrum $\operatorname{sp}_{\beta}(y)$ of $y$ with respect to $\beta$ and the spectrum $\operatorname{sp}(\beta)$ are given by

$$
s p_{\beta}(y) \equiv \bigcap_{\phi \in m_{y}} \Gamma(\phi) \text { and } s p(\beta) \equiv \bigcap_{\phi \in m} \Gamma(\phi),
$$

where $\Gamma(\phi)$ denotes the set of all $t \in G$ with $\langle\lambda(t), \phi\rangle=0$. For a closed subset $E$ of $G$ we denote by $N^{\beta}(E)$ the set of all $y$ in $N$ satisfying $s p_{\beta}(y) \subset E$. Then $N^{\beta}(\{e\})=N^{\beta}$.

Now, we shall give some properties of spectrum $s p_{\beta}(y)$ and the related ones.

LEMMA 3.1. For any $\phi$ and $\psi$ in $R(G)_{*}$ and for closed subsets $E$ and $F$ of $G$ the following eight statements hold:

(i) $s p_{\beta}\left(\beta_{\phi}(y)\right) \subset s p_{\beta}(y) \backslash \Gamma(\phi)^{\circ}$, where $\Gamma(\phi)^{\circ}$ denotes the interior of $\Gamma(\phi)$

(ii) $y \in N^{\beta}(E)$ if and only if $E^{\mathcal{U}} \subset \Gamma(\phi)$ implies $\beta_{\phi}(y)=0$ for all neighbourhood $\mathcal{U}$ of $e$ and for all $\phi \in R(G)_{*}$;

(iii) $N^{\beta}(E)$ is a weakly closed vector subspace of $N$;

(iv) $s p_{\beta}\left(x^{*}\right)=s p_{\beta}(x)^{-1}$;

(v) if $D$ is weakly total in $N$, then $\operatorname{sp}(\beta)$ is the closure of union of all $s p_{\beta}(y)$ with $y \in D$;

(vi) $t \in s p(\beta)$ if and only if $N^{\beta}(\mathcal{U}) \neq\{0\}$ for all compact neighbourhoods $\mathcal{U}$ of $t$

(vii) if $\lambda_{*} \phi=\lambda_{*} \psi$ on some neighbourhood of $\operatorname{sp}_{\beta}(y)$, then $\beta_{\phi}(y)=$ $\beta_{\psi}(y) ;$ and

(viii) if $E$ or $F$ is compact, $N^{\beta}(E) N^{\beta}(F) \subset N^{\beta}(E \cdot F)$.

Proof. (i) Put $z \equiv \beta_{\phi}(y)$. If $\beta_{\psi}(y)=0$, then $\beta_{\psi}(z)=\beta_{\phi}\left(\beta_{\psi}(y)\right)=$ 
0. Indeed, $\langle\lambda(t), \phi \psi\rangle=\langle\lambda(t), \phi\rangle\langle\lambda(t), \psi\rangle=\langle\lambda(t), \psi \phi\rangle$ and $\beta_{\psi} \beta_{\phi}=$ $\beta_{\phi \psi}=\beta_{\phi} \beta_{\psi}$ by the property of a dual action. Therefore $m_{y} \subset m_{z}$ and hence $s p_{\beta}(z) \subset s p_{\beta}(y)$.

If $t$ is in $\Gamma(\phi)^{\circ}$, there exists a $\psi$ in $R(G)_{*}$ such that $\langle\lambda(t), \psi\rangle \neq 0$ and $\phi \psi=0$. Since $\phi \psi=0$ implies $\beta_{\psi}(z)=0$, it follows that $t \notin s p_{\beta}(z)$. Therefore $s p_{\beta}(z) \subset G \backslash \Gamma(\phi)^{\circ}$.

(ii) Suppose that $y \in N^{\beta}(E)$. For any neighbourhood $\mathcal{U}$ of $e$ and $\phi$ in $R(G)_{*}$, if $E \mathcal{U} \subset \Gamma(\phi)$, then $E \subset \Gamma(\phi)^{\circ}$ and hence $E \backslash \Gamma(\phi)^{\circ}=\varnothing$. Since $s p_{\beta}\left(\beta_{\phi}(y)\right)=\varnothing$ by (i), we have $\beta_{\phi}(y)=0$.

Conversely, if $t \notin E$, there exist a neighbourhood $U$ of $e$ and a $\phi$ in $R(G)_{*}$ such that $E \mathcal{U} \subset \Gamma(\phi)$ and $\langle\lambda(t), \phi\rangle \neq 0$. From assumption it follows that $\beta_{\phi}(y)=0$. Since $\langle\lambda(t), \phi\rangle \neq 0$, we have $t \notin s p_{\beta}(y)$. Therefore $s p_{\beta}(y) \subset E$, namely, $y \in N^{\beta}(E)$.

(iii) If $y_{\kappa} \in N^{\beta}(E)$ and $y_{\kappa}$ converges weakly to $y$, then

$$
\left\langle\beta_{\phi}(y), \omega\right\rangle=\langle\beta(y), \omega \otimes \phi\rangle=\lim \left\langle\beta\left(y_{k}\right), \omega \otimes \phi\right\rangle
$$

for all $\omega \in N_{*}$. Therefore $E \mathscr{U} \subset \Gamma(\phi)$ implies $\beta_{\phi}(y)=0$, and hence $y \in N^{\beta}(E)$ by (ii).

(iv) Let $m_{t}$ denote the ideal $\{\lambda(t)\}^{\perp}$ of $R(G)_{*}$. If $t \in s p_{\beta}\left(x^{*}\right)$, then $m_{x^{*}} \subset m_{t}$. Since $\left(m_{x}\right)^{*}=m_{x^{*}}$ and $\left(m_{t}\right)^{*}=m_{t^{-1}}$, we have $m_{x} \subset m_{t^{-1}}$ and hence $t^{-1} \in s p_{\beta}(x)$. Therefore $s p_{\beta}\left(x^{*}\right) \subset s p_{\beta}(x)^{-1}$. Changing the role of $x$ and $x^{*}$, we have $s p_{\beta}(x) \subset s p_{\beta}\left(x^{*}\right)^{-1}$. Thus $s p_{\beta}\left(x^{*}\right)=s p_{\beta}(x)^{-1}$.

(v) Let $E$ be the closure of union of all $s p_{\beta}(y)$ with $y \in D$. Since $E \subset \operatorname{sp}(\beta)$ is clear, it suffices to show the converse inclusion. For this we suppose tht $s p(\beta) \backslash E$ is nonempty and $t$ is its element. For any compact neighbourhood $\mathcal{U}$ of $e$ with $t \mathscr{U} \cap E=\varnothing$ there exists a $\phi \in R(G)_{*}$ such that $\langle\lambda(t), \phi\rangle \neq 0$ and $G \backslash \Gamma(\phi)^{\circ} \subset t U$. Since

$$
s p_{\beta}(y) \backslash \Gamma(\phi)^{\circ} \subset E \cap t \mathscr{U}=\varnothing,
$$

we have $s p_{\beta}\left(\beta_{\phi}(y)\right)=\varnothing$ by (i) and so $\beta_{\phi}(y)=0$ for all $y \in D$. Since $D$ is weakly total in $N, \beta_{\phi}=0$. Since $\langle\lambda(t), \phi\rangle \neq 0$, we have $t \notin s p(\beta)$, which is a contradiction.

(vi) Suppose that $N^{\beta}(\mathcal{U}) \neq\{0\}$ for all compact neighbourhoods $\mathcal{U}$ of $t$. Then $\mathcal{U}$ has a nonempty intersection with some $s p_{\beta}(y)$ with $y \in$ $N$. Thus $t$ belongs to the closure of union of $s p_{\beta}(y)$ with $y \in N$ and hence to $s p(\beta)$ by $(v)$.

Conversely, suppose $t \in s p(\beta)$. For any compact neighbourhood $\mathcal{U}$ of $t$, there exists a $\phi \in R(G)_{*}$ such that $\langle\lambda(t), \phi\rangle \neq 0$ and $G \mid \Gamma(\phi)^{\circ} \subset$ $\mathcal{~ U}$. Since $t \in \operatorname{sp}(\beta), \beta_{\phi}(y) \neq 0$ for some $y \in N$. Since $\operatorname{sp}_{\beta}\left(\beta_{\phi}(y)\right) \subset \mathcal{U}$ by (i), it follows that $\beta_{\phi}(y) \in N^{\beta}(\mathcal{U})$.

(vii) Since $s p_{\beta}(y) \subset \Gamma(\phi-\psi)^{\circ}, \quad s p_{\beta}\left(\beta_{\phi-\psi}(y)\right)=\varnothing$ by (i). Thus $\beta_{\phi}(y)-\beta_{\psi}(y)=\beta_{\phi-\psi}(y)=0$. 
(viii) We may assume that $F$ is compact by (iv). Let $x \in N^{\beta}(E)$ and $y \in N^{\beta}(F)$. If $\omega \in N_{*}$ and $\psi \in R(G)_{*}$, then

$$
\begin{aligned}
\left\langle\Phi_{\omega}(x y), \psi\right\rangle & =\langle\beta(x) \beta(y), \omega \otimes \psi\rangle \\
& =\lim \int\left\langle\beta(x), \beta_{\lambda(r)^{*} \phi}(y) \omega \otimes \lambda(r) \psi\right\rangle d r \\
& =\lim \int\left\langle\Phi_{\left.\beta_{\lambda(r))^{*}(y) \omega}(x) \lambda(r), \psi\right\rangle d r,}\right.
\end{aligned}
$$

where the second equality is due to [17, Lemma 4.3]. Therefore

$$
\Phi_{\omega}(x y)=\lim \int z_{r} \lambda(r) d r . \quad\left(z_{r} \equiv \Phi_{\beta_{\lambda(r) \cdot \phi}(y) \omega}(x)\right)
$$

Since $s p_{\gamma}\left(z_{r} \lambda(r)\right)=s p_{\gamma}\left(z_{r}\right) r$ and since $s p_{\gamma}\left(z_{r}\right) \subset s p_{\beta}(x)$ by [17, Proposition 5.4], it follows that $s p_{\gamma}\left(z_{r} \lambda(r)\right) \subset s p_{\beta}(x) r$. If $\mathcal{U}$ is a symmetric compact neighbourhood of $e$, then $r \mapsto \beta_{\lambda(r)^{*} \phi}(y)$ vanishes on $G \backslash \mathcal{U} F$ for all $\phi$ with car $\lambda_{*} \phi \subset \mathcal{U}$. Therefore (iii) implies $s p_{\gamma}\left(\Phi_{\omega}(x y)\right) \subset s p_{\beta}(x) \mathcal{U}$, which implies (viii) by [17, Proposition 5.4].

For any projection $p$ in $N^{\beta}$ we denote by $\beta^{p}$ the restriction of $\beta$ defined by

$$
\beta^{p}\left(x_{p}\right) \equiv \beta(x)_{p \otimes 1 G} \quad x \in N,
$$

where $x_{p}$ is the restriction of $p x$ to $p \mathscr{K}$. Then $\beta^{p}$ is an isomorphism of $N_{p}$ into $N_{p} \otimes R(G)$ satisfying $\beta^{p} \otimes \iota \circ \beta^{p}=\iota \otimes \gamma \circ \beta^{p}$, that is, $\beta^{p}$ is a dual action of $G$ on $N_{p}$.

Definition 3.2. $\Gamma(\beta)$ is the intersection of all $s p \beta^{p}$, where $p$ are nonzero projections in $N^{\beta}$. $N^{\beta}$, then

LEMMA 3.3. If $p$ is a projection in $N^{\beta}$ and $q$ the central carrier of $p$ in

(i) if $N^{\beta}(E) \cap N_{q} \neq\{0\}$, then $N^{\beta}(E) \cap N_{p} \neq\{0\}$;

(ii) $s p\left(\beta^{p}\right)=s p\left(\beta^{q}\right)$; and

(iii) $\Gamma\left(\beta^{p}\right)=\Gamma\left(\beta^{q}\right)$.

Proof. (i) Let $\left(N^{\beta}\right)^{u}$ be the set of all unitaries in $N^{\beta}$. Since $q=\sup \left\{u p u^{*}: u \in\left(N^{\beta}\right)^{u}\right\}$, if $x \in N^{\beta}(E) \cap N_{q}$ and $x \neq 0$, there exist two unitaries $u$ and $v$ in $N^{\beta}$ such that $u p u^{*} x v p v^{*} \neq 0$. If we put $y \equiv$ $p u^{*} x v p$, then $y \in N_{p}, y \neq 0$ and $y \in N^{\beta}(E)$ by (viii) of Lemma 3.1.

(ii) It is clear from (vi) of Lemma 3.1 and (i). 
(iii) Since $Z\left(N_{p}^{\beta}\right)$ is isomorphic to $Z\left(N_{q}^{\beta}\right)$ by the correspondence $e_{p} \mapsto e_{q}$ for $e \in Z\left(N^{\beta}\right)$, the central carrier of $e_{p}$ in $N_{q}^{\beta}$ coincides with $e_{q}$. Since $s p \beta^{e_{p}}=s p \beta^{e_{q}}$ by (ii), $\Gamma\left(\beta^{p}\right)=\Gamma\left(\beta^{q}\right)$.

When $u$ is a weakly continuous $\alpha$ twisted unitary representation of $G$ in $M$, i.e., $u(s t) \equiv u(s) \alpha_{s}(u(t))$. Then the unitary $U$ in $M \otimes L^{\infty}(G)$ defined by $(U \xi)(r)=u(r) \xi(r)$ for $\xi \in \mathscr{H} \otimes L^{2}(G)$ satisfies

$$
U \otimes 1_{G}(\alpha \otimes \iota) U=\iota \otimes \delta(u)
$$

Proposition 3.4. If a unitary $U$ in $M \otimes L^{\infty}(G)$ satisfies (3.1), there exists a weakly continuous $\alpha$ twisted unitary representation $u$ of $G$ in $M$ such that $(U \xi)(r)=u(r) \xi(r)$ locally almost everywhere. (We can use the same letter for both $U$ and $u$.)

Proof. For $g$ in $L^{1}(G)$ we define $U(g)$ in $M$ by

$$
\langle U(g), \omega\rangle=\langle U, \omega \otimes g\rangle
$$

for all $\omega$ in $M_{*}$. If $f \in L^{1}(G)$, then

$$
\begin{aligned}
\langle U \alpha & (U(g)), \omega \otimes f\rangle=\left\langle U(g), \alpha_{*}((\omega \otimes f) U)\right\rangle \\
& =\left\langle U, \alpha_{*}((\omega \otimes f) U) \otimes g\right\rangle \quad(\text { By }(3.2)) \\
& =\left\langle U \otimes 1_{G}(\alpha \otimes \iota) U, \omega \otimes f \otimes g\right\rangle \\
& =\langle\iota \otimes \delta(U), \omega \otimes f \otimes g\rangle \quad(\text { By }(3.1)) \\
& =\langle U, \omega \otimes f * g\rangle \\
& =\int g(t)\left\langle U, \omega \otimes A d \lambda(t)^{*}(f)\right\rangle d t \\
& =\int g(t)\left\langle A d \lambda_{1}(t)(U), \omega \otimes f\right\rangle d t .
\end{aligned}
$$

Therefore

$$
U^{*} \int g(t) A d \lambda_{1}(t)(U) d t(=\alpha(U(g)))
$$

belongs to $\alpha(M)$. If we make $g(t) d t$ converge to the Dirac measure at $s$, then it converges weakly to $U^{*} A d \lambda_{1}(s)(U)$, which belongs to $\alpha(M)$. Define a unitary $u(s)$ in $M$ by

$$
u(s) \equiv \alpha^{-1}\left(U^{*} A d \lambda_{1}(s)(U)\right) .
$$


Then $s \mapsto u(s)$ is weakly continuous. Combining (3.3) and (3.4), we have

$$
U(g)=\int g(t) u(t) d t
$$

It follows from (3.2) that $(U \xi)(r)=u(r) \xi(r)$ locally almost everywhere. Besides, since $\{M, \alpha\} \sim\{\alpha(M), \iota \otimes \delta\}$ and $\alpha \circ \alpha_{t}^{\circ} \alpha^{-1}=$ $(\iota \otimes \delta)_{t}=A d \lambda_{1}(t)$, it follows from (3.4) that $u(s) \alpha_{s}(u(t))=u(s t)$.

Let $\alpha^{1}$ and $\alpha^{2}$ be two actions of $G$ on $M$. We denote by $\alpha^{1} \sim \alpha^{2}$ if there exists a unitary $u$ in $M \otimes L^{\infty}(G)$ satisfying (3.1) for $\alpha^{1}$ and $\alpha^{2}=A d u \circ \alpha^{1}$.

Definition 3.5. Let $\beta_{1}$ and $\beta_{2}$ be dual actions of $G$ on $N$. Denote by $\beta_{1} \sim \beta_{2}$ if there exists a unitary $v$ in $N \otimes R(G)$ such that

$$
v \otimes 1_{G}\left(\beta_{1} \otimes \iota\right) v=\imath \otimes \gamma(v) \text { and } \beta_{2}=A d v \circ \beta_{1}
$$

It should be noted that, when $u$ is a weakly continuous $\alpha$ twisted unitary representation of $G$ in $N, u(t) \in M^{\alpha}$ for all $t$ if and only if $\bar{\alpha}(u)=u \otimes 1_{G}$.

LEMMA 3.6. Let $\left\{e_{i j}: i, j=1,2\right\}$ be matrix units of a type $I_{2}$ factor $F_{2}$. If $\beta_{1} \sim \beta_{2}$, there exists a dual action $\beta$ of $G$ on $N \otimes F_{2}$ satisfying $\beta\left(y \otimes e_{k k}\right)=\bar{\beta}_{k}\left(y \otimes e_{k k}\right)$ for $k=1,2$, where $\bar{\beta}_{k} \equiv \iota \otimes \sigma \circ \beta_{k} \otimes \iota$ on $N \otimes F_{2}$.

Proof. Since $\beta_{1} \sim \beta_{2}$, there exists a unitary $v$ in $N \otimes R(G)$ such that $\beta_{2}=A d v \circ \beta_{1}$ and

$$
v \otimes 1_{G}\left(\beta_{1} \otimes \iota\right) v=\iota_{N} \otimes \gamma(v)
$$

If we set $w \equiv \iota_{N} \otimes \sigma\left(1 \otimes 1_{G} \otimes e_{11}+v \otimes e_{22}\right)$, then $w$ belongs to $N \otimes F_{2} \otimes R(G)$. Define a mapping $\beta$ of $N \otimes F_{2}$ into $N \otimes F_{2} \otimes R(G)$ by

$$
\begin{aligned}
& \beta\left(\Sigma y_{1 j} \otimes e_{i j}\right)=\overline{\beta_{1}}\left(y_{11} \otimes e_{11}\right)+\overline{\beta_{1}}\left(y_{12} \otimes e_{12}\right) \iota_{N} \otimes \sigma\left(v^{*} \otimes e_{22}\right) \\
& +\iota_{N} \otimes \sigma\left(v \otimes e_{22}\right) \overline{\beta_{1}}\left(y_{21} \otimes e_{21}\right)+A d\left(\iota_{N} \otimes \sigma\left(v \otimes e_{22}\right)\right)\left(\overline{\beta_{1}}\left(y_{22} \otimes e_{22}\right)\right) .
\end{aligned}
$$

Then $\beta=A d w \circ \overline{\beta_{1}}$ and hence $\beta$ is an isomorphism. Moreover, since 


$$
\begin{aligned}
& \sigma \otimes \iota \circ \iota \otimes \sigma\left(1_{G} \otimes 1_{G} \otimes e_{11}\right)=\iota_{F_{2}} \otimes \gamma \circ \sigma\left(1_{G} \otimes e_{11}\right) \\
& \iota_{N} \otimes \sigma \otimes \iota \circ \iota_{N} \otimes \iota \otimes \sigma \circ \iota_{N} \otimes \gamma \otimes \iota_{F_{2}}\left(v \otimes e_{22}\right) \\
& =\iota_{N} \otimes \iota_{F_{2}} \otimes \gamma \circ \iota_{N} \otimes \sigma\left(v \otimes e_{22}\right)
\end{aligned}
$$

it follows from (3.5) that

$$
\begin{aligned}
w \otimes & 1_{G}\left(\overline{\beta_{1}} \otimes \iota\right) w \\
= & \iota_{N} \otimes \sigma \otimes \iota\left(1 \otimes 1_{G} \otimes e_{11} \otimes 1_{G}+v \otimes e_{22} \otimes 1_{G}\right) \\
& \cdot\left(\overline{\beta_{1}} \otimes \iota_{F_{2}} \circ \iota_{N} \otimes \sigma\right)\left(1 \otimes 1_{G} \otimes e_{11}+v \otimes e_{22}\right) \\
= & \iota_{N} \otimes \sigma \otimes \iota \circ \iota_{N} \otimes \iota \otimes \sigma\left(1 \otimes 1_{G} \otimes 1_{G} \otimes e_{11}\right. \\
& \left.+v \otimes 1_{G} \otimes e_{22}\left(\beta_{1} \otimes \iota \otimes \iota_{F_{2}}\right)\left(v \otimes e_{22}\right)\right) \\
= & \iota_{N} \otimes \sigma \otimes \iota \circ \iota_{N} \otimes \iota \otimes \sigma\left(1 \otimes 1_{G} \otimes 1_{G} \otimes e_{11}\right. \\
& \left.+\iota_{N} \otimes \gamma \otimes \iota_{F_{2}}\left(v \otimes e_{22}\right)\right) \\
= & \iota_{N} \otimes \iota_{F_{2}} \otimes \gamma \circ \iota_{N} \otimes \sigma\left(1 \otimes 1_{G} \otimes e_{11}+v \otimes e_{22}\right) \\
= & \left(\iota_{N} \otimes \iota_{F_{2}} \otimes \gamma\right) w .
\end{aligned}
$$

Since $\overline{\beta_{1}}$ is a dual action of $G$ on $N \otimes F_{2}, \beta$ is also a dual action of $G$ on $N \otimes F_{2}$.

When $G$ is abelian, if $p, q \in N^{\beta}$ and $p \sim q$ in $N$, then $\Gamma\left(\beta^{p}\right)=$ $\Gamma\left(\beta^{q}\right)$. However, if $G$ is nonabelian, this is not necessarily assured.

Let $\beta_{1}$ and $\beta_{2}$ be dual actions of $G$ on $N$ such that $\beta_{2}=A d v \circ \beta_{1}$ for some $v \in N \otimes R(G)$ with $v \otimes 1_{G}\left(\beta_{1} \otimes \imath\right) v=\imath \otimes \gamma(v)$. Then we have a dual action $\beta$ of $G$ on $N \otimes F_{2}$ satisfying

$$
1 \otimes e_{j j} \in\left(N \otimes F_{2}\right)^{\beta} \quad \text { and } \quad 1 \otimes e_{11} \sim 1 \otimes e_{22}
$$

as in Lemma 3.6. Then $\Gamma\left(\beta^{1 \otimes e_{i j}}\right)=\Gamma\left(\beta_{j}\right)$ and hence $\Gamma\left(\beta_{2}\right) s p_{\beta}\left(1 \otimes e_{21}\right)=$ $s p_{\beta}\left(1 \otimes e_{21}\right) \Gamma\left(\beta_{1}\right)$.

- The condition (ii) in the following theorem is a consequence of Theorems 5.2 and 6.1, which will be proved in $\$ \S 5$ and 6 .

THEOREM 3.7. (i) $\Gamma(\beta)$ is a closed subgroup of $G$.

(ii) If $\beta$ is dual to some action (or if $\beta$ is regular), $\Gamma(\beta)$ is normal and $\Gamma(\beta)=\Gamma(\tilde{\beta})$.

Proof. (i) Since $\Gamma(\beta)$ is clearly closed, it suffices to prove the group property. Since $\Gamma(\beta)^{-1}=\Gamma(\beta)$, by (iv) of Lemma 3.1, we have only to 
show $\Gamma(\beta) \operatorname{sp} \beta^{p} \subset \operatorname{sp} \beta^{p}$ for all projections $p$ in $N^{\beta}$. Since $s p \beta^{p}$ is closed, we have only to show that, for any compact neighbourhood $u$ of $e$, $s p \beta^{p} \cap s t \mathscr{U} \neq \varnothing$ for any $s \in \Gamma(\beta)$ and $t \in s p \beta^{p}$. Choose a symmetric neighbourhood $\mathscr{V}$ of $e$ with $t^{-1} \mathscr{V} t \mathscr{V} \subset \mathcal{U}$. Since $t \in s p \beta^{p}$, there exists an element $x$ in $N$ such that $s p_{\beta}(x) \subset t \mathscr{V}$ and $x=p x p \neq 0$. Let $q$ be the carrier projection of $x^{*}$. Since $s \in \Gamma(\beta)$ and $0<q \leqq p, s \in s p \beta^{q}$ and hence there exists an element $y$ in $N$ such that $s p_{\beta}(y) \subset s \mathscr{V}$ and $y=q y q \neq 0$. Since $y x \in N, y x=p y x p \neq 0$ and $s p_{\beta}(y x) \subset$ st $\mathscr{U}$ by (viii) of Lemma 3.1, it follows that $s p \beta^{p} \cap s t \mathscr{U} \neq \varnothing$.

(ii) When $\beta$ is regular, $\Gamma(\beta)$ coincides with the set of $t \in G$ with $\hat{\beta}_{t}=\imath$ on $Z\left(N \bigotimes_{\beta}^{d} G\right)$ by Theorem 6.1. Since $Z\left(N \bigotimes_{\beta}^{d} G\right)$ is globally invariant under $\hat{\beta}$, for all $s, \Gamma(\beta)$ is normal.

If $\beta$ is regular, then $\{\bar{N}, \bar{\beta}\} \sim\{\bar{N},[\beta]\}$ and so $\Gamma(\bar{\beta})=\Gamma([\beta])=$ $\Gamma(\tilde{\beta})$. Since $\Gamma(\beta)=\Gamma(\bar{\beta})$ by Theorem 5.2, $\Gamma(\beta)=\Gamma(\tilde{\beta})$.

4. Integrable actions and dual actions. Here and hereafter, we denote the center of a given von Neumann algebra $A$ by $Z(A)$.

This section is prepared only for Theorem 8.4. First we recall a result on a weight on $M \otimes_{\alpha} G,[\mathbf{1 7}$, Section 6]. Let $\Delta$ be a semi-finite faithful normal weight on $L^{\infty}(G)$ defined by

$$
\langle f, \Delta\rangle=\int f(t) \Delta(t) d t
$$

for all $f$ in $K(G)$. Here, we need no fair of notational confusion for $\Delta$. Let $n_{\alpha}$ be the set of all $x$ in $M$ satisfying

$$
\left|\left\langle\alpha\left(x^{*} x\right), \omega \otimes \Delta\right\rangle\right| \leqq \lambda_{x}\|\omega\| \quad\left(\lambda_{x}>0\right)
$$

for all $\omega$ in $M_{*}$. Then there exists a faithful normal $M^{\alpha}$ valued weight $E_{\alpha}$ on $M$, whose domain is $\mathfrak{n}_{\alpha}^{*} n_{\alpha}$.

In case of compact $G$ it is straightforward that

(a) $\Delta$ is bounded (it may be assumed a state);

(b) $E_{\alpha}^{2}=E_{\alpha}$ and $\left\|E_{\alpha}\right\|=1$ (if $\langle 1, \Delta\rangle=1$ );

(c) $M^{\alpha} \subset \mathrm{n}_{\alpha}$; and

(d) $\mathbf{n}_{\alpha}$ is $\sigma$-weakly dense in $M$.

However, for a noncompact $G$, the assertions (a), (b) and (c) do not hold. Following Connes and Takesaki [6] we call $\alpha$ to be integrable, if $E_{\alpha}$ is semi-finite, namely, $\alpha$ satisfies (d). It is shown in [17] that the action $\hat{\beta}$ dual to some $\beta$ is always integrable. Since $\left(\pi_{\alpha}\right)_{p} \subset \pi^{\alpha^{p}}$ for $p \in M^{\alpha}$, if $\alpha$ is integrable, so is $\alpha^{p}$. 
THEOREM 4.1. (Connes and Takesaki). Assume that $G$ is separable. If $M^{\alpha}$ is properly infinite, the following three conditions are equivalent:

(i) $\quad \alpha$ is integrable;

(ii) for each non zero projection $f$ in $M^{\alpha}$ there is an $x$ in $\bar{M}$ satisfying $q x q \neq 0\left(q \equiv f \otimes 1_{G}\right)$ and

$$
\left(1 \otimes V^{\prime}\right) \bar{\alpha}(q x q)=q x q \otimes 1_{G}
$$

(iii) $\{\bar{M}, \bar{\alpha}\} \sim\left\{\bar{M}_{p},[\alpha]^{p}\right\}$ for some projection $p$ in $\bar{M}^{[\alpha]}$.

Proof. (i) $\Rightarrow$ (ii) Since $\alpha$ is integrable by assumption (i), there exists a nonzero $z$ in $\mathfrak{n}_{\alpha}$ with $z=f z f$. For any $g$ in $K(G)$ we set

$$
(x \xi)(s) \equiv \Delta(s)^{1 / 2} \alpha_{s}(z) \eta, \quad \eta \equiv \int g(t) \xi(t) d t
$$

for $\xi$ in $\mathscr{H} \otimes L^{2}(G)$. Then $q x q=\left(f \otimes 1_{G}\right) x\left(f \otimes 1_{G}\right)=x$. Since $z \neq 0$, $x \neq 0$. Since

$$
\begin{aligned}
\|x \xi\|^{2} & =\int\left\|\alpha_{\mathrm{s}}(z) \eta\right\|^{2} \Delta(s) d s=\left\langle E_{\alpha}\left(z^{*} z\right), \omega_{\eta}\right\rangle \\
& \leqq\left\|E_{\alpha}\left(z^{*} z\right)\right\|\|\eta\|^{2} \leqq\left\|E_{\alpha}\left(z^{*} z\right)\right\|\|g\|_{2}^{2}\|\xi\|_{2}^{2},
\end{aligned}
$$

$x$ is bounded on $\mathscr{H} \otimes L^{2}(G)$. If we replace $\xi$ by $\left(x^{\prime} \otimes 1_{G}\right) \xi$ with $x^{\prime} \in M^{\prime}$ in (4.2), then $\left[x, x^{\prime} \otimes 1_{G}\right]=0$ and so $x \in \vec{M}$. Since

$$
\begin{aligned}
((1 & \left.\left.\otimes V^{\prime}\right) \bar{\alpha}(x) \xi\right)(s, t)=\Delta(t)^{1 / 2}(\bar{\alpha}(x) \xi)\left(t^{-1} s, t\right) \\
\quad & =\Delta(t)^{1 / 2} \bar{\alpha}_{t}(x) \xi\left(t^{-1} s, t\right) \\
& =\Delta(t)^{1 / 2} \Delta\left(t^{-1} s\right)^{1 / 2} \alpha_{t}\left(\alpha_{t^{-}{ }_{s}}(z)\right) \int g(r) \xi(r, t) d r \\
& =\Delta(s)^{1 / 2} \alpha_{s}(z) \int g(r) \xi(r, t) d r \\
& =\left(\left(x \otimes 1_{G}\right) \xi\right)(s, t)
\end{aligned}
$$

we have $q x q(=x)$ satisfies the equality in (ii).

(ii) $\Rightarrow$ (iii) It suffices to show that the set $I_{\alpha}$ of $x$ in $\bar{M}$ with $\left(1 \otimes V^{\prime}\right) \bar{\alpha}(x)=x \otimes 1_{G}$ contains an isometry $v$. Indeed, $v v^{*} \in \bar{M}^{[\alpha]}$ and $A d v \otimes 1_{G} \circ \bar{\alpha}=[\alpha] \circ A d v$.

The case where $M^{\alpha}$ is $\sigma$-infinite. Since

$$
1 \otimes V^{\prime} \otimes 1_{G}(\bar{\alpha} \otimes \iota)\left(1 \otimes V^{\prime}\right)=\iota_{\bar{M}} \otimes \delta\left(1 \otimes V^{\prime}\right),
$$


we can define an action $\langle\alpha\rangle$ of $G$ on $\bar{M} \otimes F_{2}$ by

$$
\begin{aligned}
\langle\alpha\rangle\left(\sum x_{\imath \jmath} \otimes e_{\imath \jmath}\right)= & \overline{[\alpha]}\left(x_{11} \otimes e_{11}\right) \\
& +\overline{[\alpha]}\left(x_{12} \otimes e_{12}\right) \iota_{\bar{M}} \otimes \sigma\left(1 \otimes V^{\prime} \otimes e_{22}\right) \\
& +\iota_{\bar{M}} \otimes \sigma\left(1 \otimes V^{\prime *} \otimes e_{22}\right) \overline{[\alpha]}\left(x_{21} \otimes e_{21}\right) \\
& +\iota_{\bar{M}} \otimes \sigma \circ \bar{\alpha} \otimes \iota_{F_{2}}\left(x_{22} \otimes e_{22}\right),
\end{aligned}
$$

where $\overline{[\alpha]} \equiv \iota_{\bar{M}} \otimes \sigma \circ[\alpha] \otimes \iota_{F_{2}}$. Then $\Sigma x_{i j} \otimes e_{i j} \in\left(\bar{M} \otimes F_{2}\right)^{\langle\alpha\rangle}$ if and only if

$$
x_{11} \in \bar{M}^{[\alpha]}, \quad x_{12} \in I_{\alpha}, \quad x_{21} \in I_{\alpha}^{*} \quad \text { and } \quad x_{22} \in \bar{M}^{\bar{\alpha}} .
$$

Therefore the central carrier of $1_{\bar{M}} \otimes e_{22}$ in $\left(\bar{M} \otimes F_{2}\right)^{\langle\alpha\rangle}$ is majorized by the central carrier of $1_{\bar{M}} \otimes e_{11}$ by (ii). Since $M^{\alpha}$ is properly infinite and $G$ is separable by assumption, $1_{\bar{M}} \otimes e_{11}$ is properly infinite and $1_{\bar{M}} \otimes e_{22}$ is $\sigma$-infinite in $\left(\bar{M} \otimes F_{2}\right)^{\langle\alpha\rangle}$. Therefore $1_{\bar{M}} \otimes e_{22}<1_{\bar{M}} \otimes e_{11}$ in $\left(\bar{M} \otimes F_{2}\right)^{\langle\alpha\rangle}$ from the above discussion. Thus there exists a partial isometry $v \otimes e_{12}$ in $\left(\bar{M} \otimes F_{2}\right)^{\langle\alpha\rangle}$ whose initial and final projections are $1_{\bar{M}} \otimes e_{22}$ and the one majorized by $1_{\bar{M}} \otimes e_{11}$, respectively. Then $v$ is an isometry in $I_{\alpha}$.

The general case. There exists a partition $\left\{e_{\iota}: \iota \in I\right\}$ in $Z\left(M^{\alpha}\right)$ satisfying either

(a) $e_{\iota}$ is $\sigma$-finite in $M^{\alpha}$; or

(b) $e_{\iota}$ is divided into uncountable set $\left\{f_{\kappa}: \kappa \in K\right\}$ of mutually equivalent, properly infinite and $\sigma$-infinite projections in $M^{\alpha}$. Since $M^{\alpha}$ is properly infinite, $e_{\imath}$ with (a) is also properly infinite in $M^{\alpha}$. Thus we can apply the above $\sigma$-finite case of $M^{\alpha}$ to $\left\{M_{e_{\iota}}, \alpha^{e}\right\}$ or $\left\{M_{f_{\kappa}}, \alpha^{f_{\kappa}}\right\}$.

(iii) $\Rightarrow$ (i) Since $\tilde{\alpha}$ is dual to $\hat{\alpha},[\alpha]$ is integrable. If $p \in \bar{M}^{[\alpha]}$ is nonzero, $[\alpha]^{p}$ is also integrable on $\bar{M}_{p}$. Therefore $\bar{\alpha}$ is integrable on $\bar{M}$ by our assumption (iii). Let $q$ be a projection in $\bar{M}$ of the form $1 \otimes e$ for some minimal $e$ in $B\left(L^{2}(G)\right)$. Since $q \in \bar{M}^{\bar{\alpha}}, \bar{\alpha}^{q}$ is integrable on $\bar{M}_{q}$. Since $\{M, \alpha\}$ is equivalent to $\left\{\bar{M}_{q}, \bar{\alpha}^{q}\right\}, \alpha$ is integrable on $M$.

Now we consider the dual version. Let $\omega_{e}$ be the semi-finite faithful normal weight on $R(G)$ defined by

$$
\left\langle\lambda\left(\Delta^{1 / 2}\left(\tilde{g}_{*} g\right)\right), \omega_{e}\right\rangle=\tilde{g}_{*} g(e), \quad \tilde{g}(t) \equiv \overline{g\left(t^{-1}\right)}
$$

for $g$ in $K(G)$ and $\mathfrak{n}_{\beta}$ the set of all $y$ in $N$ satisfying

$$
\left|\left\langle\beta\left(y^{*} y\right), \omega \otimes \omega_{e}\right\rangle\right| \leqq \lambda_{y}\|\omega\|
$$


for all $\omega$ in $N_{*}$. There exists a faithful normal $N^{\beta}$ valued weight $E_{\beta}$ on $N$ with domain $\mathfrak{n}_{\beta}^{*} \mathfrak{n}_{\beta}$. When $G$ is discrete,

(a) $\omega_{e}$ is bounded (it may be assumed a state);

(b) $E_{\beta}^{2}=E_{\beta}$ and $\left\|E_{\beta}\right\|=1$ (if $\left\langle 1, \omega_{e}\right\rangle=1$ );

(c) $N^{\beta} \subset n_{\beta}$; and

(d) $\mathfrak{n}_{\beta}$ is $\sigma$-weakly dense in $N$.

However, for a nondiscrete $G,(\mathrm{a}),(\mathrm{b})$ and (c) do not hold, and moreover,

(e) $\left(N^{\beta}(\{s\}) \cap \pi_{\beta}\right) \backslash\{0\}=\varnothing$ for all $s$.

Definition 4.2. $\quad \beta$ is integrable if for any nonzero projection $f$ in $N^{\beta}$ there is an element $y$ in $\bar{N}$ satisfying $q y q \neq 0\left(q \equiv f \otimes 1_{G}\right)$ and

$$
(1 \otimes W) \bar{\beta}(q y q)=q y q \otimes 1_{G} \text {. }
$$

THEOREM 4.3. Assume that $G$ is separable and $N^{\beta}$ is properly infinite. If $\beta$ is integrable, then

$$
\{\bar{N}, \bar{\beta}\} \sim\left\{\bar{N}_{p},[\beta]^{p}\right\}
$$

for some projection $p$ in $\bar{N}^{[\beta]}$.

Proof. We denote by $I_{\beta}$ the set of all $y$ in $\bar{N}$ satisfying

$$
(1 \otimes W) \bar{\beta}(y)=y \otimes 1_{G} .
$$

Since $\bar{\beta}\left(y^{*} y\right)=y^{*} y \otimes 1_{G}, \quad q_{0} \equiv \sup \left\{s\left(y^{*} y\right): \quad y \in I_{\beta}\right\} \quad$ belongs to $\bar{N}^{\bar{\beta}}$, where $s(y * y)$ is the carrier of $y * y$. Since $I_{\beta} u=I_{\beta}$ for $u$ in $\bar{N}^{\bar{\beta}}$, $q_{0} \in Z\left(\bar{N}^{\bar{\beta}}\right)$. Since $\beta$ is integrable, $q_{0}=1$. Since

$$
1 \otimes W \otimes 1_{G}(\bar{\beta} \otimes \iota)(1 \otimes W)=\iota_{N} \otimes \gamma(1 \otimes W),
$$

we can define a dual action $\langle\beta\rangle$ of $G$ on $\bar{N} \otimes F_{2}$ by

$$
\begin{aligned}
\langle\beta\rangle\left(\Sigma y_{i j} \otimes e_{i j}\right)= & \overline{[\beta]}\left(y_{11} \otimes e_{11}\right) \\
& +\overline{[\beta]}\left(y_{12} \otimes e_{12}\right) \iota_{\bar{N}} \otimes \sigma\left(1 \otimes W \otimes e_{22}\right) \\
& +\iota_{\bar{N}} \otimes \sigma\left(1 \otimes W^{*} \otimes e_{22}\right) \overline{[\beta]}\left(y_{21} \otimes e_{21}\right) \\
& +\iota_{\bar{N}} \otimes \sigma \circ \bar{\beta} \otimes \iota\left(y_{22} \otimes e_{22}\right)
\end{aligned}
$$

as shown in Lemma 3.6. Then $\Sigma y_{\imath \jmath} \otimes e_{\iota \jmath} \in\left(\bar{N} \otimes F_{2}\right)^{\langle\beta\rangle}$ if and only if

$$
y_{11} \in \bar{N}^{[\beta]}, \quad y_{12} \in I_{\beta}, \quad y_{21} \in I_{\beta}^{*} \quad \text { and } \quad y_{22} \in \bar{N}^{\bar{\beta}} \text {. }
$$


Since $G$ is separable and $N^{\beta}$ is properly infinite, $1_{\bar{N}} \otimes e_{22}<1_{\bar{N}} \otimes e_{11}$ in $\left(\bar{N} \otimes F_{2}\right)^{(\beta)}$ from the above. Therefore there exists an isometry $w$ in $I_{\beta}$. Consequently, (4.4) implies

$$
A d w \otimes 1_{G} \circ \bar{\beta}=[\beta] \circ A d w .
$$

Since $[\beta]\left(w w^{*}\right)=w w^{*} \otimes 1_{G}$ by $(4.3), p \equiv w w^{*}$ belongs to $\bar{N}^{[\beta]}$ and $\{\bar{N}, \bar{\beta}\} \sim\left\{\bar{N}_{p},[\beta]^{p}\right\}$.

5. Regular actions and dual actions. In this section we shall discuss the relation between a (dual) action dual to some one and a dominant (dual) action. The former is initially characterized by Landstad and the latter concept is initially given by Connes and Takesaki. For our convenience we shall introduce the following terminology for $\alpha$ and $\beta$.

Definition 5.1. An action $\alpha$ (resp. a dual action $\beta$ ) of $G$ on $M$ (resp. $N$ ) is regular if there exists a covariant dual system $\{N, \beta\}$ (resp. covariant system $\{M, \alpha\}$ ) satisfying $\{M, \alpha\} \sim\left\{N \bigotimes_{\beta}^{d} G, \hat{\beta}\right\}$ (resp. $\{N, \beta\} \sim$ $\left.\left\{M \otimes_{\alpha} G, \hat{\alpha}\right\}\right)$.

Therefore, $\beta$ is regular if and only if there exists a weakly continuous unitary representation $u$ of $G$ in $N$ such that $\beta(u(t))=u(t) \otimes \lambda(t)$ for all $t$; and $\alpha$ is regular if and only if there exists an isomorphism $\pi$ of $L^{\infty}(G)$ into $M$ such that $\alpha_{t}^{\circ} \pi=\pi \circ A d \lambda^{\prime}(t)$ for all $t,[15 ; 16 ; 17$, Theorems 8.1 and $8.3 ; 21]$. It is immediate from our definition that $\hat{\alpha}, \hat{\beta}$, $\tilde{\alpha}, \tilde{\beta},[\alpha]$ and $[\beta]$ are regular.

The ergodicity of $\alpha$ (resp. $\beta$ ) on $Z(M)$ (resp. $Z(N))$ is defined by $M^{\alpha} \cap Z(M)=\mathrm{C} 1\left(\right.$ resp. $\left.N^{\beta} \cap Z(N)=\mathrm{C} 1\right)$.

THEOREM 5.2. (i) $Z\left(M \bigotimes_{\alpha} G\right) \subset \alpha(M)$ (resp. $\left.Z\left(N \bigotimes_{\beta}^{d} G\right) \subset \beta(N)\right)$ if and only if $Z\left(\tilde{M} \otimes_{\tilde{\alpha}} G\right) \subset \tilde{\alpha}(\tilde{M})$ (resp. $Z\left(\tilde{N} \otimes_{\tilde{\beta}}^{d} G\right) \subset \tilde{\beta}(\tilde{N})$.

(ii) $\alpha$ (resp. $\beta)$ is ergodic on $Z(M)($ resp. $Z(N))$ if and only if $\tilde{\alpha}$ (resp. $\tilde{\beta})$ is ergodic on $Z(\tilde{M})$ (resp. $Z(\tilde{N}))$.

(iii) $\operatorname{Ker} \alpha\lceil Z(M)=\operatorname{Ker} \tilde{\alpha} \backslash Z(\tilde{M})$, where $\operatorname{Ker} \alpha\lceil Z(M)$ is the set of all $t$ in $G$ with $\alpha_{t}=\imath$ on $Z(M)$.

(iv) $\Gamma(\beta)=\Gamma(\bar{\beta})$.

Proof. Our proof owes to Takesaki's duality:

$$
\{\tilde{M}, \tilde{\alpha}\} \sim\{\bar{M},[\alpha]\} \quad \text { and } \quad\{\tilde{N}, \tilde{\beta}\} \sim\{\bar{N},[\beta]\} .
$$

(i) The case of $\alpha . \quad$ Let $N \equiv M \otimes_{\alpha} G$ and $\beta \equiv \hat{\alpha} . \quad$ According to (5.1) we have only to show the equivalence of $Z(N) \subset N^{\beta}$ and $Z(\bar{N}) \subset$ 
$\bar{N}^{[\beta]}$. Since $Z(\bar{N})=Z(N) \otimes 1_{G}$, we have only to show that $y \in N^{\beta}$ if and only if $y \otimes 1_{G} \in \bar{N}^{[\beta]}$ for all $y \in Z(N)$. However, this is clear from the following equality

$$
A d 1 \otimes W \circ \iota \otimes \sigma \circ \beta \otimes \iota\left(y \otimes 1_{G}\right)=y \otimes 1_{G} \otimes 1_{G} .
$$

The case of $\beta$. Let $M \equiv N \bigotimes_{\beta}^{d} G$ and $\alpha \equiv \hat{\beta}$. According to (5.1) we have only to show the equivalence of $Z(M) \subset M^{\alpha}$ and $Z(\bar{M}) \subset$ $\bar{M}^{[\alpha]}$. Since $Z(\bar{M})=Z(M) \otimes 1_{G}$, we have only to show that $x \in M^{\alpha}$ if and only if $x \otimes 1_{G} \in \bar{M}^{[\alpha]}$ for all $x \in Z(M)$. This is clear from

$$
\operatorname{Ad} 1 \otimes V^{\prime} \circ \iota \otimes \sigma \circ \alpha \otimes \iota\left(x \otimes 1_{G}\right)=x \otimes 1_{G} \otimes 1_{G} .
$$

(ii) The case of $\alpha$. Since $\alpha(x)=x \otimes 1_{G}$ if and only if $[\alpha]\left(x \otimes 1_{G}\right)=$ $x \otimes 1_{G} \otimes 1_{G}$, it follows that $\alpha$ is ergodic on $Z(M)$ if and only if $[\alpha]$ is ergodic on $Z(M) \otimes 1_{G}$. Thus we have (ii) for $\alpha$ by (5.1).

The case of $\beta$. Since $\beta(y)=y \otimes 1_{G}$ if and only if $[\beta]\left(y \otimes 1_{G}\right)=$ $y \otimes 1_{G} \otimes 1_{G}$, both of the ergodicity of $\beta$ on $Z(N)$ and $[\beta]$ on $Z(N) \otimes 1_{G}$ are equivalent. Thus we have (ii) for $\beta$ by (5.1).

(iii) It is immediate from (5.1) that

$$
\operatorname{Ker} \alpha\lceil Z(M)=\operatorname{Ker}[\alpha]\lceil Z(\bar{M})=\operatorname{Ker} \tilde{\alpha}\lceil Z(\tilde{M}) .
$$

(iv) If $y \in N, z \in B\left(L^{2}(G)\right), \omega_{1} \in N_{*}, \omega_{2} \in B\left(L^{2}(G)\right)_{*}$ and $\phi \in$ $R(G)_{*}$, then

$$
\left\langle\bar{\beta}_{\phi}(y \otimes z), \omega_{1} \otimes \omega_{2}\right\rangle=\left\langle\beta_{\phi}(y) \otimes z, \omega_{1} \otimes \omega_{2}\right\rangle
$$

and hence $\beta_{\phi}=0$ is equivalent to $\bar{\beta}_{\phi}=0$. Since $Z\left(\bar{N}^{\bar{\beta}}\right)=Z\left(N^{\beta}\right) \otimes 1_{G}$, it follows that $s p \beta^{p}=s p \bar{\beta}^{p \otimes 1_{G}}$ for $p \in Z\left(N^{\beta}\right)$. Consequently, $\Gamma(\beta)=$ $\Gamma(\bar{\beta})$.

When $M$ is properly infinite and $M$ contains a partition $\left\{e_{\iota}: \iota \in I\right\}$ of the identity such that $e_{\iota} \sim e_{\kappa}$ in $M$ for all $\iota, \kappa$ and $\operatorname{dim} L^{2}(G) \leqq$ Card $I$, Takesaki's duality tells us that $\left(M \bigotimes_{\alpha} G\right) \bigotimes_{\alpha}^{d} G$ is isomorphic to $M$. Here we raise a question, when is $\left\{\left(M \bigotimes_{\alpha} G\right) \bigotimes_{\tilde{\alpha}}^{d} G, \tilde{\alpha}\right\}$ equivalent to $\{M, \alpha\}$ ?, namely, when are they isomorphic as a covariant system?

We shall begin with the following lemma.

LEMMA 5.3. If $M^{\alpha}$ (resp. $\left.N^{\beta}\right)$ contains a partition $\left\{e_{\iota}: \iota \in I\right\}$ of the identity satisfying

(i) $e_{\iota} \sim 1$ in $M^{\alpha}$ (resp. $N^{\beta}$ ) for all $\iota \in I$; and

(ii) $\operatorname{dim} H \leqq$ Card $I$, then $\{M, \alpha\}$ (resp. $\{N, \beta\}$ ) is spatially isomorphic to 
Proof. From our assumptions (i) and (ii) we can obtain a partition $\left\{f_{\iota}: \iota \in I_{H}\right\}$ of the identity such that $\operatorname{Card} I_{H}=\operatorname{dim} H$ and $f_{\iota} \sim 1$ in $M^{\alpha}$ (resp. $N^{\beta}$ ) for all $\iota \in I_{H}$. Let $v_{\iota}$ be an isometry in $M^{\alpha}$ (resp. $N^{\beta}$ ) with $v_{\imath} v_{\iota}^{*}=f_{\iota}$. Let $\rho$ be the spatial isomorphism of $M$ (resp. $N$ ) onto $M \otimes B(H)$ (resp. $N \otimes B(H)$ ) such that

$$
\rho(x)=\sum_{\iota, \kappa} v_{\iota}^{*} x v_{\kappa} \otimes e_{\iota \kappa}
$$

for $x$ in $M$ (resp. $N$ ), where $\left\{e_{\iota \kappa}: \iota, \kappa \in I_{H}\right\}$ is the set of matrix units of $B(H)$. Then

$$
\begin{aligned}
& \rho\left(\alpha_{t}(x)\right)=\sum v_{\imath}^{*} \alpha_{t}(x) v_{\kappa} \otimes e_{\iota \kappa} \\
&=\sum \alpha_{t}\left(v_{\imath}^{*} x v_{\kappa}\right) \otimes e_{\iota \kappa}=\left(\alpha_{t} \otimes \iota\right)(\rho(x)) \\
&(\operatorname{resp.}(\rho \otimes \iota) \beta(x)=(\iota \otimes \sigma \circ(\iota \otimes \sigma \circ \rho \otimes \iota)) \beta(x) \\
&=\iota \otimes \sigma\left(\sum \beta\left(v^{*} x v_{k}\right) \otimes e_{\iota \kappa}\right)=(\iota \otimes \sigma \circ \beta \otimes \iota) \rho(x),
\end{aligned}
$$

where the second equality follows from the following reason. If $s p_{\beta}(x)$ is compact, then, by [17, Lemma 4.3],

$$
\left.\left(v_{\imath}^{*} \otimes 1_{G}\right) \beta(x)\left(v_{\kappa} \otimes 1_{G}\right)=\lim _{\phi} \int \beta_{\lambda(r)^{*} \phi}\left(v^{*} x v_{k}\right) \otimes \lambda(r) d r\right) .
$$

An action $\alpha$ (resp. a dual action $\beta$ ) is said to be of infinite multiplicity if it satisfies the conditions (i) and (ii) for a Hilbert space $H \equiv L^{2}(G)$ and an infinite $G$ in Lemma 5.3.

Definition 5.4. An action $\alpha$ (resp. a dual action $\beta$ ) of $G$ on $M$ (resp. $N$ ) is said to be dominant if

(i) $\alpha$ (resp. $\beta$ ) is of infinite multiplicity, and

(ii) $\{\bar{M}, \bar{\alpha}\} \sim\{\bar{M},[\alpha]\}$ (resp. $\{\bar{N}, \bar{\beta}\} \sim\{\bar{N},[\beta]\}$ ).

For a dominant $\alpha$ (resp. $\beta$ ) it holds that

$$
\{M, \alpha\} \sim\{\tilde{M}, \tilde{\alpha}\} \quad(\operatorname{resp} .\{N, \beta\} \sim\{\tilde{N}, \tilde{\beta}\})
$$

by Lemma 5.3 .

REMARK 5.5. If $G$ is infinite and $\alpha$ (resp. $\beta$ ) is regular, $\bar{\alpha}(\operatorname{resp} . \bar{\beta})$ is dominant. 
THEOREM 5.6. If $\alpha$ (resp. $\beta$ ) is regular, then

$$
\{\bar{M}, \bar{\alpha}\} \sim\{\bar{M},[\alpha]\} \quad(\text { resp. }\{\bar{N}, \bar{\beta}\} \sim\{\bar{N},[\beta]\}) .
$$

Proof. The case of $\{M, \alpha\}$. If $\alpha$ is regular, there exists a covariant dual system $\{N, \beta\}$ satisfying

$$
\{M, \alpha\} \sim\left\{N \otimes_{\beta}^{d} G, \hat{\beta}\right\} .
$$

Let $\mathscr{K}$ denote the underlying Hilbert space of $N$. Since

$$
\begin{aligned}
& \hat{\beta}_{t}(\beta(y))=\beta(y)=A d \lambda_{l}^{\prime}(t)(\beta(y)) \\
& \hat{\beta}_{t}\left(1_{\mathscr{H}} \otimes f\right)=1_{\mathscr{H}} \otimes f_{t^{-1}}=A d \lambda_{1}^{\prime}(t)\left(1_{\mathscr{K}} \otimes f\right)
\end{aligned}
$$

for $\lambda_{i}^{\prime}(t) \equiv 1_{\mathscr{K}} \otimes \lambda^{\prime}(t)$, we have $\hat{\beta}_{t}=A d \lambda_{1}^{\prime}(t)$ on $N \otimes_{\beta}^{d} G$. Let $w$ be the unitary on $\mathscr{K} \otimes L^{2}(G) \otimes L^{2}(G)$ defined by

$$
\left(1_{\mathscr{K}} \otimes W^{*}\right)\left(1_{\mathscr{K}} \otimes 1_{G} \otimes J\right) \text {. }
$$

Since $A d W\left(\lambda^{\prime}(r) \otimes 1_{G}\right)=\lambda^{\prime}(r) \otimes \lambda(r)$, it follows that

$$
w^{*} A d \lambda_{1}^{\prime}(r) \otimes 1_{G}(w)=1_{\Re} \otimes 1_{G} \otimes \lambda^{\prime}(r) .
$$

Therefore, by (5.4)

$$
(A d w)^{-1} \circ \hat{\beta}_{t} \otimes \iota \circ A d w=\hat{\beta}_{t} \otimes A d \lambda^{\prime}(t)
$$

on $\left(N \bigotimes_{\beta}^{d} G\right) \otimes B\left(L^{2}(G)\right)$, which completes the proof for $\alpha$.

The case of $\{N, \beta\}$. If $\beta$ is regular, there exists a weakly continuous unitary representation $u$ of $G$ in $N$ satisfying $\beta(u(t))=$ $u(t) \otimes \lambda(t)$. We denote by the same letter $u$ the unitary in $N \otimes L^{\infty}(G)$ defined by $(u \xi)(r)=u(r) \xi(r)$ for $\xi \in \mathscr{K} \otimes L^{2}(G)$. Then $(1 \otimes W) \bar{\beta}\left(u^{*}\right)=u^{*} \otimes 1_{G}$. Indeed, if $\xi \in \mathscr{K} \otimes L^{2}(G) \otimes L^{2}(G)$, then

$$
\begin{aligned}
((u & \left.\left.\otimes 1_{G}\right)(1 \otimes W) \xi\right)(s, t)=u(s) \xi(s, t s) \\
& =u(s)((1 \otimes U) \xi)(t s, s) \\
& =\beta(u(s)) \otimes 1_{G}((1 \otimes U) \xi)(t, s) \\
& =((\beta \otimes \iota(u))(1 \otimes U) \xi)(t, s) \\
& =((1 \otimes U)(\beta \otimes \iota(u))(1 \otimes U) \xi(s, t) \\
& =(\bar{\beta}(u) \xi)(s, t),
\end{aligned}
$$

where $U$ is a unitary on $L^{2}(G) \otimes L^{2}(G)$ defined by $(U \eta)(s, t) \equiv \eta(t, s)$ for all $\eta$ in $L^{2}(G) \otimes L^{2}(G)$. Therefore 


\section{$A d u \otimes 1 \circ A d 1 \otimes W \circ \bar{\beta}=\bar{\beta} \circ A d u$}

and hence $\{\bar{N},[\beta]\} \sim\{\bar{N}, \bar{\beta}\}$.

It should be noted that the proof of Theorem 5.6 for $\beta$ does not use the homomorphism property of $u$.

COROllaRy 5.7. $\alpha$ (resp. $\beta$ ) is dominant if and only if it is regular and of infinite multiplicity.

6. Ergodic actions and dual actions. This section is devoted to further investigation of [6, $\S 3$ of Chapter III]. The following theorem generalizes [6, Theorem III, 3.2], whose proof will go along the same line of argument. By Lemma 3.3

$$
\Gamma(\beta)=\cap\left\{s p \beta^{p}: p \in Z\left(N^{\beta}\right), p \neq 0\right\} .
$$

For a given action $\alpha$ we denote by $\operatorname{Ker} \alpha\lceil Z(M)$ the set of all $t$ in $G$ satisfying $\alpha_{t}=\iota$ on $Z(M)$.

THEOREM 6.1. (i) $\Gamma(\beta)=\operatorname{Ker} \hat{\beta} \mid Z\left(N \bigotimes_{\beta}^{d} G\right)$ if $\beta$ is regular.

(ii) $\Gamma(\hat{\alpha})=\operatorname{Ker} \alpha\lceil Z(M)$.

Proof. (i) If $\beta$ is regular, there exists an isomorphism $\rho$ of $N^{\beta} \otimes_{\alpha} G$ onto $N$ satisfying $\beta \circ \rho=\rho \otimes \iota \circ \hat{\alpha}$, where $\alpha$ is an action of $G$ on $N^{\beta}$. Then Takesaki's duality gives us that

$$
\left\{N \bigotimes_{\beta}^{d} G, \hat{\beta}\right\} \sim\left\{N^{\beta} \otimes B\left(L^{2}(G)\right),[\alpha]\right\} .
$$

Therefore

(6.2) $\operatorname{Ker} \hat{\beta}\left\lceil Z\left(N \bigotimes_{\beta}^{d} G\right)=\operatorname{Ker}[\alpha] \mid Z\left(N^{\beta}\right) \otimes 1_{G}=\operatorname{Ker} \alpha\left\lceil Z\left(N^{\beta}\right)\right.\right.$.

Since $\left\{N^{\beta}, \alpha\right\}$ is covariant and $\rho \circ \alpha\left(N^{\beta}\right)=N^{\beta}$ by (2.7), we have

$$
\alpha_{t}^{\circ} \equiv(\rho \circ \alpha) \circ \alpha_{t} \circ(\rho \circ \alpha)^{-1} \in \text { Aut } N^{\beta} .
$$

Here we set $u(t) \equiv \rho(1 \otimes \lambda(t))$. Since $\beta(u(t))=u(t) \otimes \lambda(t), N^{\beta}(\{t\})=$ $N^{\beta} u(t)$ by [17, Proposition 5.2]. If $p$ is a nonzero projection in $Z\left(N^{\beta}\right)$, then $\alpha_{t}^{\circ}(p)=u(t) p u(t)^{*}$ and

$$
p N^{\beta}(\{t\}) p=p N^{\beta} u(t) p=p N^{\beta} \alpha_{t}^{\circ}(p) u(t) .
$$


Consequently, $s \in \operatorname{Ker} \hat{\beta} \backslash Z\left(N \bigotimes_{\beta}^{d} G\right)$ if and only if $\alpha_{s}=\iota$ on $Z\left(N^{\beta}\right)$ by (6.2), if and only if $\alpha_{s}^{\circ}=\iota$ on $Z\left(N^{\beta}\right)$, if and only if $p \alpha_{s}^{\circ}(p) \neq 0$ for all nonzero projection $p$ in $Z\left(N^{\beta}\right)$, if and only if $s \in \Gamma(\beta)$ by (6.1) and (6.3).

(ii) Combining (i) and Theorem 5.2, we have

$$
\Gamma(\hat{\alpha})=\operatorname{Ker} \tilde{\alpha}\lceil Z(\tilde{M})=\operatorname{Ker} \alpha\lceil Z(M) .
$$

Here we denote the von Neumann algebra generated by $\beta(N)$ and $1 \otimes\left(L^{\infty}(G) \cap \lambda^{\prime}(H)^{\prime}\right)$ by $N \bigotimes_{\beta}^{d}(H \backslash G)$, whose characterization will be discussed in $\S 7$.

Corollary 6.2. Assume $\beta$ is regular. For a closed subgroup $H$ of $G, \Gamma(\beta)=H$ if and only if $H$ is the largest subgroup satisfying $Z\left(N \bigotimes_{\beta}^{d} G\right) \subset N \bigotimes_{\beta}^{d}(H \backslash G)$. In particular, $\Gamma(\beta)=G$ if and only if $Z\left(N \otimes_{\beta}^{d} G\right) \subset \beta(N)$.

Proof. $\Gamma(\beta)=H$ if and only if $H$ is the largest subgroup satisfying the condition that $\hat{\beta}_{t}=\iota$ on $Z\left(N \bigotimes_{\beta}^{d} G\right)$ for all $t \in H$ by Theorem 6.1. The condition is equivalent to

$$
Z\left(N \bigotimes_{\beta}^{d} G\right) \subset\left\{x \in N \bigotimes_{\beta}^{d} G: \hat{\beta}_{t}(x)=x, t \in H\right\}=N \bigotimes_{\beta}^{d}(H \backslash G)
$$

by Theorem 7.2 in $\$ 7$.

Here, if we combine Theorem 5.2 and Corollary 6.2, we have that $\Gamma(\tilde{\beta})=G$ if and only if $Z\left(N \bigotimes_{\beta}^{d} G\right) \subset \beta(N)$.

From our previous result [14, Proposition 3.1] we have the following proposition.

Proposition 6.3. If $\alpha$ (resp. $\beta$ ) is regular, the following two conditions are equivalent:

(i) $Z\left(M \otimes_{\alpha} G\right) \subset \alpha(M)($ resp. $\Gamma(\beta)=G)$; and

(ii) $Z\left(M^{\alpha}\right) \subset Z(M)$ (resp. $\left.Z\left(N^{\beta}\right) \subset Z(N)\right)$.

If $\alpha$ (resp. $\beta)$ in the above is ergodic on $Z(M)($ resp. $Z(N))$, then $M^{\alpha}$ (resp. $N^{\beta}$ ) is a factor.

Proof. The case of $\{M, \alpha\}$. If $\alpha$ is regular, there exists a dual action $\beta$ of $G$ on $M^{\alpha}$ such that

$$
\{M, \alpha\} \sim\left\{N \bigotimes_{\beta}^{d} G, \hat{\beta}\right\},
$$

where $N \equiv M^{\alpha}$.

(i) $\Rightarrow$ (ii) Since $Z\left(M \bigotimes_{\alpha} G\right) \subset \alpha(M)$ by (i) and $\alpha(M)=\left(M \bigotimes_{\alpha} G\right)^{\hat{\alpha}}$, it follows from (6.4) that 


$$
Z(N) \otimes 1_{G}=Z\left(N \otimes B\left(L^{2}(G)\right)\right) \subset\left(N \otimes B\left(L^{2}(G)\right)\right)^{[\beta]} .
$$

If $z \in Z(N)$, then $[\beta]\left(z \otimes 1_{G}\right)=z \otimes 1_{G} \otimes 1_{G}$ and hence

$$
\beta \otimes \iota\left(z \otimes 1_{G}\right)=\left(\iota \otimes \sigma \circ A d 1 \otimes W^{*} \circ[\beta]\right)\left(z \otimes 1_{G}\right)=z \otimes 1_{G} \otimes 1_{G} .
$$

Therefore $\beta(z)=z \otimes 1_{G}$ for $z \in Z(N)$. Since $\beta(z)$ commutes with $\beta(N)$ and $1 \otimes L^{\infty}(G), \beta(z)$ belongs to $Z\left(N \otimes_{\beta}^{d} G\right)$, which shows that $\beta(Z(N)) \subset Z\left(N \otimes_{\beta}^{d} G\right)$. Consequently, (6.4) implies that $Z\left(M^{\alpha}\right) \subset$ $Z(M)$, because $\beta(N)=\left(N \bigotimes_{\beta}^{d} G\right)^{\hat{\beta}}$.

(ii) $\Rightarrow$ (i) We apply Takesaki's duality to (6.4) and we have

$$
\left\{M \otimes_{\alpha} G, \hat{\alpha}\right\} \sim\left\{N \otimes B\left(L^{2}(G)\right),[\beta]\right\}
$$

If we can show that

$$
Z(N) \otimes 1_{G} \subset N \otimes_{\beta}^{d} G
$$

then $Z\left(M \otimes_{\alpha} G\right) \subset\left(M \otimes_{\alpha} G\right)^{\hat{\alpha}}=\alpha(M)$ by (6.5). Therefore we want to show (6.6).

Now, since $\beta$ is a dual action of $G$ on $N$, we have

$$
\{N, \beta\} \sim\{\beta(N), \iota \otimes \gamma\}
$$

and $(\iota \otimes \gamma) \beta(y)=A d 1 \otimes W^{*}\left(\beta(y) \otimes 1_{G}\right)$. According to our assumption $Z\left(M^{\alpha}\right) \subset Z(M)$, we have

$$
Z((\iota \otimes \gamma) \beta(N)) \subset Z\left(\beta(N) \otimes_{\iota \otimes \gamma}^{d} G\right) \subset\left(\beta(N) \otimes_{\iota \otimes \gamma}^{d} G\right)^{\prime}
$$

by (6.4) and (6.7). Therefore $(\iota \otimes \gamma) \beta(Z(N))$ commutes with $1 \otimes 1_{G} \otimes L^{\infty}(G)$ and hence $\beta(Z(N)) \otimes 1_{G}$ commutes with Ad $1 \otimes W\left(1 \otimes 1_{G} \otimes L^{\infty}(G)\right)$. Since $Z(\beta(N)) \otimes 1_{G}$ commutes with $\beta(N)^{\prime} \otimes 1_{G}$, we have

$$
Z(\beta(N)) \otimes 1_{G} \subset\left\{\beta(N)^{\prime} \otimes 1_{G}, \text { Ad } 1 \otimes W\left(1 \otimes 1_{G} \otimes L^{\infty}(G)\right)\right\}^{\prime},
$$

the right hand side of which is $\beta(N) \otimes_{\imath}^{d} \otimes_{\gamma} G$ by [16, Theorem 5]. Therefore, (6.6) is proved by (6.7).

The case of $\{N, \beta\}$. If $\beta$ is regular, there exists an action $\alpha$ of $G$ on $N^{\beta}$ such that

$$
\{N, \beta\} \sim\left\{M \otimes_{\alpha} G, \hat{\alpha}\right\}
$$

where $M \equiv N^{\beta}$. 
(i) $\Rightarrow$ (ii) Since $\operatorname{Ker} \hat{\beta} \mid Z\left(N \bigotimes_{\beta}^{d} G\right)=\Gamma(\beta)=G$ by Theorem 6.1 and (i), $\hat{\beta}_{t}=\imath$ on $Z\left(N \otimes_{\beta}^{d} G\right)$ and hence $[\alpha]_{t}=\imath$ on $Z\left(M \otimes B\left(L^{2}(G)\right)\right)$ by (6.8). Therefore $\alpha_{t}=\imath$ on $Z(M)$ for all $t$ and so $\alpha(z)=z \otimes 1_{G}$ for $z \in Z(M)$. Since $\alpha(z) \in M \otimes_{\alpha} G$ and it commutes with $\alpha(M)$ and $1 \otimes R(G), \quad \alpha(z)$ belongs to $Z\left(M \otimes_{\alpha} G\right), \quad$ namely, $\quad Z(\alpha(M)) \subset$ $Z\left(M \otimes_{\alpha} G\right)$. Consequently, (6.8) implies that $Z\left(N^{\beta}\right) \subset Z(N)$, because $\alpha(M)=\left(M \otimes_{\alpha} G\right)^{\hat{\alpha}}$.

(ii) $\Rightarrow$ (i) Since $\beta$ is regular, there is a weakly continuous unitary representation $u$ of $G$ in $N$ such that $\beta(u(t))=u(t) \otimes \lambda(t)$. Since $Z\left(N^{\beta}\right) \subset Z(N)$ by (ii), if $p$ is a nonzero projection in $Z\left(N^{\beta}\right), \beta^{p}$ is a dual action of $G$ on $N_{p}$ and $t \mapsto u(t)_{p}$ is a weakly continuous unitary representation of $G$ in $N_{p}$ satisfying $\beta^{p}\left(u(t)_{p}\right)=$ $u(t)_{p} \otimes \lambda(t)$. Therefore $s p \beta^{p}=G$ for all $p$ in $Z\left(N^{\beta}\right)$. Consequently, $\Gamma(\beta)=G$.

Finally, if $\alpha$ (resp. $\beta$ ) is ergodic on $Z(M)$ (resp. $Z(N)$ ), then $M^{\alpha}$ (resp. $N^{\beta}$ ) is a factor from the above.

THEOREM 6.4. The following two conditions are equivalent:

(i) $\quad M \otimes_{\alpha} G$ (resp. $N \bigotimes_{\beta}^{d} G$ ) is a factor; and

(ii) $Z\left(M \otimes_{\alpha} G\right) \subset \alpha(M)($ resp. $\Gamma(\tilde{\beta})=G)$ and $\alpha$ (resp. $\left.\beta\right)$ is ergodic on $Z(M)$ (resp. $Z(N))$.

Proof. The case of $\{M, \alpha\}$. (i) $\Rightarrow$ (ii) If $\alpha(z)=z \otimes 1_{G}$ for $z \in Z(M), \alpha(z)$ commutes with $\alpha(M)$ and $1 \otimes R(G)$, and hence it belongs to $Z\left(M \otimes_{\alpha} G\right)$. Since $M \otimes_{\alpha} G$ is a factor, (ii) follows immediately.

(ii) $\Rightarrow$ (i) We denote the covariant system $\left\{\left(M \otimes_{\alpha} G\right) \bigotimes_{\hat{\alpha}} G, \hat{\hat{\alpha}}\right\}$ by $\{\tilde{M}, \tilde{\alpha}\}$. Since $\hat{\alpha}\left(M \otimes_{\alpha} G\right)=\tilde{M}^{\tilde{\alpha}}$, it suffices to show that $\tilde{M}^{\tilde{\alpha}}$ is a factor. Since $\tilde{\alpha}$ is regular, it suffices to show that

(a) $Z\left(\tilde{M} \otimes_{\tilde{\alpha}} G\right) \subset \tilde{\alpha}(\tilde{M})$; and

(b) $\tilde{\alpha}$ is ergodic on $Z(\tilde{M})$

by Proposition 6.3. (a) and (b) are immediate from (ii) by Theorem 5.2.

The case of $\{N, \beta\}$. (i) $\Rightarrow$ (ii) Since $\Gamma(\tilde{\beta})=G$ is clear, it suffices to show the ergodicity of $\beta$ on $Z(N)$. If $\beta(z)=z \otimes 1_{G}$ for $z \in Z(N)$, then $\beta(z)$ commutes with $\beta(N)$ and $1 \otimes L^{\infty}(G)$, and hence $\beta(z)$ belongs to $Z\left(N \otimes_{\beta}^{d} G\right)$. Since $N \otimes_{\beta}^{d} G$ is a factor by (i), $\beta$ is ergodic on $Z(N)$.

(ii) $\Rightarrow$ (i) Let $\quad\{\tilde{N}, \tilde{\beta}\} \equiv\left\{\left(N \bigotimes_{\beta}^{d} G\right) \bigotimes_{\hat{\beta}} G, \hat{\beta}\right\}$. Since $\hat{\beta}\left(N \bigotimes_{\beta}^{d} G\right)=$ $\tilde{N}^{\tilde{\beta}}$, it suffices to show that $\tilde{N}^{\tilde{\beta}}$ is a factor. Since $\tilde{\beta}$ is regular, it suffices to note that

(c) $\Gamma(\tilde{\beta})=G$; and

(d) $\tilde{\beta}$ is ergodic on $Z(\tilde{N})$

by Proposition 6.3. (d) is clear from (ii) by Theorem 5.2. 
COROLlaRY 6.5. If $\alpha$ (resp. $\beta$ ) is regular and if $Z\left(M \otimes_{\alpha} G\right) \subset \alpha(M)$ (resp. $\Gamma(\beta)=G)$, then $\alpha^{p}\left(\right.$ resp. $\left.\beta^{p}\right)$ is regular for all $p \in Z\left(M^{\alpha}\right)$ (resp. $\left.Z\left(N^{\beta}\right)\right)$.

Proof. The case of $\alpha$. Since $\alpha$ is regular, $\{M, \alpha\}$ is identified with $\left\{N \otimes_{\beta}^{d} G, \hat{\beta}\right\}$ for some $\{N, \beta\}$. Since $\hat{\beta}_{t}(\beta(y))=\beta(y)$ and $\hat{\beta}_{t}(1 \otimes f)=$ $1 \otimes f_{t^{-1}}$, it follows that $\hat{\beta}_{t}=A d \lambda_{1}^{\prime}(t)$ on $N \otimes_{\beta}^{d} G$. Since $Z\left(M^{\alpha}\right) \subset Z(M)$ by Proposition 6.3, $p$ belongs to $B(\mathscr{K}) \otimes L^{\infty}(G)$, where $\mathscr{K}$ is the underlying Hilbert space of $N$. An action $t \mapsto A d \lambda_{1}^{\prime}(t)$ on $B(\mathscr{K}) \otimes L^{\infty}(G)$ coincides with $\hat{\beta}_{t}$ on $Z\left(M^{\alpha}\right)$. Since $\hat{\beta}_{t}(p)=p$, $\operatorname{Ad} \lambda_{1}^{\prime}(t)(p)=p$. Since $L^{\infty}(G) \cap R(G)=\mathbf{C} 1_{G}, p$ is of the form $e \otimes 1_{G}$ for some projection $e$ in $B(\mathscr{K})$. Let $\pi$ be an isomorphism of $L^{\infty}(G)$ into $M_{p}$ defined by $\pi(f) \equiv e \otimes f\left(\in M_{p}\right)$. Then $\pi$ satisfies $\alpha_{t} \circ \pi=$ $\pi \circ A d \lambda^{\prime}(t)$ on $L^{\infty}(G)$. Therefore $\alpha^{p}$ is regular on $M_{p}$.

The case of $\beta$. Since $\beta$ is regular, there exists a weakly continuous unitary representation $u$ of $G$ in $N$ such that $\beta(u(t))=$ $u(t) \otimes \lambda(t)$. Since $Z\left(N^{\beta}\right) \subset Z(N)$ by Proposition 6.3, $t \mapsto u(t)_{p}$ is a weakly continuous unitary representation of $G$ in $N_{p}$ and hence $\beta^{p}$ is a regular dual action of $G$ on $N_{p}$.

LEMMA 6.6. Let $p$ be a projection in $M$. If

(i) $\alpha$ is regular and $Z(M) \subset M^{\alpha}$; and

(ii) $p$ is properly infinite, then $p \otimes 1_{G} \sim \alpha(p)$ in $M \otimes L^{\infty}(G)$.

Proof. Since $\alpha$ is regular by (i), $\{M, \alpha\}$ is identified with $\left\{N \otimes_{\beta}^{d} G, \hat{\beta}\right\}$ for some covariant dual system $\{N, \beta\}$. Then $\alpha(x)=$ Ad $1_{N} \otimes V^{\prime}\left(x \otimes 1_{G}\right)$ for $x$ in $M$. Since

$$
A d 1_{N} \otimes V^{\prime}(1 \otimes f)=1 \otimes f, \quad\left(1 \equiv 1_{M}=1_{N} \otimes 1_{G}\right)
$$

$M \otimes L^{\infty}(G)$ is globally invariant under $A d 1_{N} \otimes V^{\prime}$ by Lemma 8.2. Let $\rho$ be the restriction of $A d 1_{N} \otimes V^{\prime}$ to $M \otimes L^{\infty}(G)$. Then the condition (i) implies that $\rho=\iota$ on $Z(M) \otimes L^{\infty}(G)$ by (6.8). Therefore $p \otimes 1_{G} \sim$ $\rho(p)(=\alpha(p))$ by Suzuki's Theorem [N. Suzuki, Tôhoku Math. J. 7 (1955), 186-191, Theorem 1]*.

THEOREM 6.7. If

(i) $G$ is separable;

(ii) $\beta$ is integrable and $\Gamma(\tilde{\beta})=G$; and

(iii) $N^{\beta}$ is properly infinite, then $\{\overline{\bar{N}}, \iota \otimes \sigma \circ \bar{\beta} \otimes \iota\} \sim\{\overline{\bar{N}},[\bar{\beta}]\}$, where $\overline{\bar{N}} \equiv \bar{N} \otimes B\left(L^{2}(G)\right)$. 
Proof. According to (i), (ii) and (iii) we have

$$
\{\bar{N}, \bar{\beta}\} \sim\left\{\bar{N}_{p},[\beta]^{p}\right\}
$$

for some $p$ in $\bar{N}^{[\beta]}$ by Theorem 4.3. Since $N$ is properly infinite by (iii), so is $p$. Let $q$ be the central carrier of $p$ in $\bar{N}^{\{\beta\}}$. Since

$$
Z\left(\bar{N} \otimes_{[\beta]}^{d} G\right) \subset[\beta](\bar{N})
$$

by (ii) and Theorem 5.2, it follows from Corollary 6.5 that $[\beta]^{a}$ is regular. Therefore we can identify $\left\{\bar{N}_{q},[\beta]^{q}\right\}$ with $\left\{M \otimes_{\alpha} G, \hat{\alpha}\right\}$ for some covariant system $\{M, \alpha\}$. Since $Z\left(N^{[\beta]}\right) \subset Z(\bar{N})$ by Proposition 6.3, (6.10) implies that $Z\left(\bar{N}_{q} \otimes_{[\beta]^{q}}^{d} G\right)$ is included in $[\beta]^{q}(\bar{N})$ and hence $Z(\bar{M}) \subset \bar{M}^{[\alpha]}$. Therefore, if $z \in Z(M)$, then $[\alpha]\left(z \otimes 1_{G}\right)=$ $z \otimes 1_{G} \otimes 1_{G}$ and hence $\alpha(z)=z \otimes 1_{G}$. Namely, $Z(M) \subset M^{\alpha}$ or $Z(\alpha(M))=M^{\alpha} \otimes 1_{G}$.

Now we apply Lemma 6.6 to the covariant system $\{\alpha(M), \iota \otimes \delta\}$ and the projection $p \in \alpha(M)$. Then we have a partial isometry $v$ in $\alpha(M) \otimes L^{\infty}(G) \quad$ satisfying $\quad v v^{*}=p \otimes 1_{G} \quad$ and $\quad v^{*} v=\imath \otimes \delta(p)=$ $\alpha \otimes \iota(p)$. As $1_{M} \otimes V$ is a function $t \mapsto 1_{M} \otimes \lambda(t) \quad$ in $\left(1_{M} \otimes R(G)\right) \otimes L^{\infty}(G)$ and

$$
\begin{gathered}
v V_{M}\left(v V_{M}\right)^{*}=v v^{*}=p \otimes 1_{G} \quad\left(V_{M} \equiv 1_{M} \otimes V\right) \\
\left(v V_{M}\right)^{*} v V_{M}=V_{M}^{*} \alpha \otimes \iota(p) V_{M}=p \otimes 1_{G},
\end{gathered}
$$

it follows that $u \equiv\left(v V_{M}\right)_{p \otimes 1 G}$ is a unitary in $\bar{N}_{p} \otimes L^{\infty}(G)$ and

$$
[\beta]^{p}(u(t))=u(t) \otimes \lambda(t) .
$$

Therefore the note given after Theorem 5.6 gives our desired results.

7. Subgroups and subalgebras.. Throughout this section $H, d_{H}$ and $\Delta_{H}$ denote a closed subgroup of $G$, the right invariant Haar measure and the modular function, respectively. We define a subalgebra of $M \otimes_{\alpha} G$ (resp. $N \bigotimes_{\beta}^{d} G$ ) associated with $H$ by

$$
\begin{aligned}
M \otimes_{\alpha} H & \equiv\{\alpha(M), 1 \otimes \lambda(H)\}^{\prime \prime} \\
\text { (resp. } N \bigotimes_{\beta}^{d}(H \backslash G) & \equiv\left\{\beta(N), 1 \otimes\left(L^{\infty}(G) \cap \lambda^{\prime}(H)^{\prime}\right\}^{\prime \prime}\right) .
\end{aligned}
$$

Utilizing $\hat{\alpha}$ (resp. $\hat{\beta}$ ), we shall give a correspondence between a subgroup of $G$ and a subalgebra of $M \bigotimes_{\alpha} G$ (resp. $N \bigotimes_{\beta}^{d} G$ ) of the form (7.1) (resp. (7.2)), which generalizes a result due to Takesaki, [23, Theorems 7.2 and 
7.3]. For the notational convenience we set

$$
\mathscr{L}^{\infty}(G / H) \equiv L^{\infty}(G) \cap \lambda(H)^{\prime} \quad \text { and } \quad \mathscr{L}^{\infty}(H \backslash G) \equiv L^{\infty}(G) \cap \lambda^{\prime}(H)^{\prime}
$$

in what follows.

THEOREM 7.1. let $N \equiv M \otimes_{\alpha} G$ and $\beta \equiv \hat{\alpha}$.

(i) $M \otimes_{\alpha} H=\left\{y \in N: \beta(y) \in N \otimes \lambda(H)^{\prime \prime}\right\}$

$$
=N \cap\left(1 \otimes \mathscr{L}^{\infty}(G / H)\right)^{\prime} \text {. }
$$

(ii) $H$ coincides with the smallest closed subgroup $H^{\prime}$ of $G$ satisfying $\beta\left(M \otimes_{\alpha} H\right) \subset N \otimes \lambda\left(H^{\prime}\right)^{\prime \prime}$.

Proof. (i) Since

$$
\beta(\alpha(x))=\alpha(x) \otimes 1_{G} \quad \text { and } \quad \beta\left(\lambda_{1}(t)\right)=\lambda_{1}(t) \otimes \lambda(t),
$$

it follows that $\beta\left(M \otimes_{\alpha} H\right) \subset N \otimes \lambda(H)^{\prime \prime}$.

Next we shall show that $\beta(y) \in N \otimes \lambda(H)^{\prime \prime}$ implies $y \in$ $\left(1 \otimes \mathscr{L}^{\infty}(G / H)\right)^{\prime}$ for $y \in N$. For this we have only to show

$$
\mathscr{L}^{\infty}(G / H) \otimes 1_{G} \subset\left\{A d W\left(1_{G} \otimes \lambda(H)^{\prime}\right) \cup 1_{G} \otimes B\left(L^{2}(G)\right)\right\}^{\prime \prime} .
$$

Indeed, $\quad y \otimes 1_{G} \quad$ commutes with $\quad A d 1 \otimes W\left(N^{\prime} \otimes \lambda(H)^{\prime}\right) \quad$ and $1_{N} \otimes B\left(L^{2}(G)\right)$ by assumption and hence $y$ commutes with $1 \otimes \mathscr{L}^{\infty}(G / H)$ by (7.3). Now, if $f \in \mathscr{L}^{\infty}(G / H) \cap C(G), \quad \delta^{c} f \in$ $\mathscr{L}^{\infty}(G / H) \otimes L^{\infty}(G)$, where $\left(\delta^{c} f\right)(s, t) \equiv f(t s)$. For any $g, h \in K(G)$ with $\|\Delta g\|_{1}=1$ we set

$$
F_{g, h} \equiv\left(\int\left(\delta^{c} f_{r^{-1}}\right)\left(1_{G} \otimes g_{r^{-1}}\right) d r\right)\left(1_{G} \otimes h\right) .
$$

Since $f \in \lambda(H)^{\prime}$ and $\delta^{c} f=A d W\left(1_{G} \otimes f\right), F_{g, h}$ belongs to the right hand side of (7.3). If $\xi \in L^{2}(G) \otimes L^{2}(G)$, then

$$
\left(F_{g, h} \xi\right)(s, t)=\left(\int f\left(r^{-1} s\right) g\left(r^{-1}\right) d r\right) \Delta(t)^{-1} h(t) \xi(s, t) .
$$

Since $r \mapsto f\left(r^{-1} s\right)$ is continuous and bounded, if $g\left(r^{-1}\right) d r$ converges to the Dirac measure at the unit $e$, then $F_{g, h}$ converges weakly to $f \otimes\left(\Delta^{-1} h\right)$. Therefore $f \otimes\left(\Delta^{-1} h\right)$ belongs to the right hand side of (7.3). Making $\Delta^{-1} h$ converge weakly to $1_{G}$, we have the inclusion (7.3) for $\mathscr{L}^{\infty}(G / H) \cap C(G)$ instead of $\mathscr{L}^{\infty}(G / H)$. Since $\mathscr{L}^{\infty}(G / H) \cap C(G)$ is weakly dense in $\mathscr{L}^{\infty}(G / H)$, we have (7.3).

Finally we shall show $M \otimes_{\alpha} H=N \cap\left(1 \otimes \mathscr{L}^{x}(G / H)\right)^{\prime}$. Our proof will go along the same line as Takesaki's proof. Suppose that $y \in N \cap$ $\left(1 \otimes \mathscr{L}^{\infty}(G / H)\right)^{\prime}$. We may assume that $M$ is standard. Let $J$ be the 
modular conjugation operator of $M \otimes_{\alpha} G$. Since $J=v\left(J_{M} \otimes J_{G}\right)=$ $\left(J_{M} \otimes J_{G}\right) v$ for a certain weakly continuous unitary representation $v$ of $G$ in $M$, we have

$$
J\left(1 \otimes \mathscr{L}^{\infty}(G / H)\right)^{\prime} J=\left(1 \otimes \mathscr{L}^{\infty}(H \backslash G)\right)^{\prime}
$$

and hence $J y J \in N^{\prime} \cap\left(1 \otimes \mathscr{L}^{\infty}(H \backslash G)\right)^{\prime}$. Here we apply the modified Blattner-Mackey's theorem for induced covariance representations [22, Theorem 4.3]. There exists a natural isomorphism $\rho$ of $\left(M \otimes_{\alpha \mid H} H\right)^{\prime}$ onto $N^{\prime} \cap\left(1 \otimes \mathscr{L}^{\infty}(H \backslash G)\right)^{\prime}$ such that

$$
\rho\left(x^{\prime} \otimes 1_{H}\right)=x^{\prime} \otimes 1_{G} \quad \text { and } \quad \rho\left(u(t) \otimes \lambda_{H}^{\prime}(t)\right)=u(t) \otimes \lambda^{\prime}(t)
$$

for $x^{\prime} \in M^{\prime}$ and $t \in H$, where $u$ is a strongly continuous unitary representation of $G$ on $\mathscr{H}$ implementing $\alpha$ and where $\lambda_{H}^{\prime}$ is the left regular representation of $H$ on $L^{2}(H)$. Therefore $J y J$ belongs to $\rho\left(\left(M \otimes_{\alpha\lceil H} H\right)^{\prime}\right)$, which is generated by $x^{\prime} \otimes 1_{G}$ and $u(t) \otimes \lambda^{\prime}(t)$ for $x^{\prime} \in M^{\prime}$ and $t \in H$. Since $v(t) u(t)^{*} \in M^{\prime}$ and since

$$
J\left(x^{\prime} \otimes 1_{G}\right) J=\alpha\left(J_{M} x^{\prime} J_{M}\right)
$$

and

$$
J\left(v(t) \otimes \lambda^{\prime}(t)\right) J=1 \otimes \lambda(t)
$$

it follows that $y$ belongs to $M \otimes_{\alpha} H$.

(ii) It is clear from the first equality in (i).

In the above proof we have established a bijective correspondence of a closed subgroup $H$ and a subalgebra $\mathscr{L}^{\infty}(G / H)$ by the relation

$$
\mathscr{L}^{\infty}(G / H)^{\prime}=\left\{x \in B\left(L^{2}(G)\right): A d W^{*}\left(x \otimes 1_{G}\right) \in B\left(L^{2}(G)\right) \otimes \lambda(H)^{\prime \prime}\right\} .
$$

Indeed, since $\mathscr{L}^{\infty}(G / H)^{\prime}$ is generated by $L^{x}(G)$ and $\lambda(H)$, it is included in the right hand side. The converse inclusion is direct from (7.3).

The dual version of Theorem 7.1 is the following.

THEOREM 7.2. Let $M \equiv N \bigotimes_{\beta}^{d} G$ and $\alpha \equiv \hat{\beta}$.

(i) $N \bigotimes_{\beta}^{d}(H \backslash G)=\left\{x \in M: \alpha_{t}(x)=x, t \in H\right\}$

$$
=M \cap\left(1 \otimes \lambda^{\prime}(H)\right)^{\prime} \text {. }
$$

(ii) $H=\left\{t \in G: \alpha_{t}(x)=x, x \in N \bigotimes_{\beta}^{d}(H \backslash G)\right\}$.

Proof. (i) Since the action $\alpha$ dual to $\beta$ is defined by $\alpha(z)=$ Ad $1 \otimes V^{\prime}\left(z \otimes 1_{G}\right)$ for $z \in N \otimes_{\beta}^{d} G$, we have 


$$
\begin{aligned}
\alpha_{t} & (z) \xi(s, t)=(\alpha(z) \xi)(s, t) \\
& =\left(\left(1 \otimes V^{\prime}\right)\left(z \otimes 1_{G}\right)\left(1 \otimes V^{\prime}\right)^{*} \xi\right)(s, t) \\
& =\lambda_{1}^{\prime}(t) z\left(\left(1 \otimes V^{\prime}\right)^{*} \xi\right)(s, t) \\
& =\lambda_{1}^{\prime}(t) z \lambda_{1}^{\prime}(t)^{*} \xi(s, t),
\end{aligned}
$$

where $\lambda_{i}^{\prime}(t) \equiv 1 \otimes \lambda^{\prime}(t)$. Therefore the second equality in (i) is proved. Moreover, $\alpha_{t}=A d \lambda_{1}^{\prime}(t)$ on $N \otimes_{\beta}^{d} G$.

Now we shall show the first equality. Put $\alpha_{t}^{H} \equiv \alpha_{t}$ for all $t \in H . \quad \alpha^{H}$ is then an action of $H$ on $N \bigotimes_{\beta}^{d} G$. Since $N \bigotimes_{\beta}^{d}(H \backslash G)$ is clearly included in $\left(N \otimes_{\beta}^{d} G\right)^{\alpha^{H}}$, it remains to show the inclusion relation

$$
N_{0} \equiv\left(N \bigotimes_{\beta}^{d}(H \backslash G)\right)^{\prime} \subset\left(\left(N \bigotimes_{\beta}^{d} G\right)^{\alpha^{H}}\right)^{\prime} .
$$

According to Theorem 1.1 we have

$$
\left(N_{0}\right)^{\beta^{H}} \subset\left(\beta(N)^{\prime}\right)^{\beta^{\prime}}=\left(N \bigotimes_{\beta}^{d} G\right)^{\prime} \subset\left(\left(N \bigotimes_{\beta}^{d} G\right)^{\alpha^{H}}\right)^{\prime}
$$

where $\beta^{H}$ is defined by (7.6) below. Moreover, $1 \otimes \lambda^{\prime}(H)$ commutes with $\left(N \otimes_{\beta}^{d} G\right)^{\alpha^{H}}$. Therefore Lemma 7.3 below implies (7.5).

(ii) Let $H_{0}$ be the set of all $t$ such that $\alpha_{t}(x)=x$ for all $x \in N \otimes_{\beta}^{d}(H \backslash G)$. Then $H_{0}$ is a closed subgroup of $G$. Since $H \subset H_{0}$ and $N \bigotimes_{\beta}^{d}(H \backslash G) \subset N \bigotimes_{\beta}^{d}\left(H_{0} \backslash G\right)$ by (i), we have $H=H_{0}$.

Lemma 7.3. If $\beta^{H}$ is a mapping defined on $\left(N \bigotimes_{\beta}^{d}(H \backslash G)\right)^{\prime}$ by

$$
\beta^{H}(y) \equiv A d 1 \otimes W\left(y \otimes 1_{G}\right),
$$

then (i) $\beta^{H}$ is a dual action of $G$ on $\left(N \bigotimes_{B}^{d}(H \backslash G)\right)^{\prime}$; and

(ii) $\left(N \bigotimes_{\beta}^{d}(H \backslash G)\right)^{\prime}=\left\{\left(\left(N \bigotimes_{\beta}^{d}(H \backslash G)\right)^{\prime}\right)^{\beta^{H}}, 1 \otimes \lambda^{\prime}(H)\right\}^{\prime \prime}$.

Proof. (i) Since $\left[W, 1_{G} \otimes \lambda^{\prime}(t)\right]=0$ and

$$
\begin{aligned}
& A d 1 \otimes W^{*}\left(\beta(N) \otimes 1_{G}\right) \subset \beta(N) \otimes R(G) \\
& A d 1 \otimes W^{*}\left(1 \otimes \mathscr{L}^{\infty}(H \backslash G) \otimes 1_{G}\right)=1 \otimes \mathscr{L}^{\infty}(H \backslash G) \otimes 1_{G},
\end{aligned}
$$

we have $\beta^{H}\left(N_{0}\right) \subset N_{0} \otimes R(G)$, where $N_{0} \equiv\left(N \otimes_{\beta}^{d}(H \backslash G)\right)^{\prime}$. Since $W \otimes 1_{G}$ satisfies (2.5), $\beta^{H}$ is a dual action of $G$ on $N_{0}$.

(ii) As $N_{0} \subset\left(1 \otimes \mathscr{L}^{\infty}(H \backslash G)\right)^{\prime}, \quad \beta^{H}\left(N_{0}\right) \quad$ is included in $N_{0} \otimes \lambda(H)^{\prime \prime}$. Let $\rho$ be an isomorphism of $\lambda(H)^{\prime \prime}$ onto $\lambda_{H}(H)^{\prime \prime}$ with $\rho(\lambda(t))=\lambda_{H}(t)$ and let $\beta_{H} \equiv \iota \otimes \rho \circ \beta^{H}$, where $\lambda_{H}$ is the right regular representation of $H$. Then $\beta_{H}$ is a dual action of $H$ on $N_{0}$. Since $1 \otimes \lambda^{\prime}(H) \subset N_{0}$ and $\beta_{H}\left(1 \otimes \lambda^{\prime}(r)\right)=1 \otimes \lambda^{\prime}(r) \otimes \lambda_{H}(r), N_{0}$ is generated 
by $\left(N_{0}\right)^{\beta_{H}}$ and $1 \otimes \lambda^{\prime}(H)$ by a characterization of a crossed product due to Landstad. Since $\left(N_{0}\right)^{\beta^{H}}=\left(N_{0}\right)^{\beta_{H}}$, we complete the proof.

COROLlaRY 7.4. There exists a semi-finite faithful normal operator valued weight $E$ on $N \bigotimes_{\beta}^{d} G$ onto $N \bigotimes_{\beta}^{d}(H \backslash G)$.

Proposition 7.5. Let $\alpha$ be an action of $G$ on $M$ and $H$ a closed subgroup of $G$. Then $H$ is normal if and only if $M \otimes_{\alpha} H$ is invariant under $A d \lambda_{1}(t)$ for all $t$.

Proof. Since

$$
A d \lambda_{1}(t)(\alpha(x))=\alpha\left(\alpha_{t}(x)\right), \quad A d \lambda_{1}(t)\left(\lambda_{1}(s)\right)=\lambda_{1}\left(t s t^{-1}\right),
$$

if $H$ is normal, $M \otimes_{\alpha} H$ is invariant under $A d \lambda_{1}(t)$. On the other hand, if $H$ is nonnormal, $M \otimes_{\alpha} H$ is not invariant.

8. Galois correspondence. In this section we shall give a Galois correspondence between closed subgroups of $G$ and globally $\beta$ invariant von Neumann subalgebras of $N$ containing $N^{\beta}$ in Theorem 8.4, which generalizes [6, Theorem III.4.3]. If $L_{0}$ is an $\alpha$ invariant subalgebra, then $\alpha\left(L_{0}\right) \subset L_{0} \otimes L^{\infty}(G)$, and vice versa. Therefore a von Neumann subalgebra $L$ of $N$ is said to be $\beta$ invariant if $\beta(L) C$ $L \otimes R(G)$. In such cases $\alpha\left\lceil L_{0}\right.$ and $\beta\left\lceil L\right.$ are an action of $G$ on $L_{0}$ and a dual action of $G$ on $L$, respectively.

THEOREM 8.1. Let $\alpha$ be an action of $G$ on $M$ and $L$ a von Neumann subalgebra with $\alpha(M) \subset L \subset M \otimes_{\alpha} G$. If $M$ is a factor, then the following two conditions are equivalent:

(i) $L$ is $\hat{\alpha}$ invariant; and

(ii) there exists a closed subgroup $H$ of $G$ such that $L=M \otimes_{\alpha} H$ (or $\left.L=\left\{y \in M \otimes_{\alpha} G: \hat{\alpha}(y) \in\left(M \bigotimes_{\alpha} G\right) \otimes \lambda(H)^{\prime \prime}\right\}\right)$.

Before going into the proof we shall prepare the following lemma, which is implicitly proved in the proof of [17, Theorem 3.1].

$$
\text { LeMmA 8.2. } M \otimes L^{\infty}(G)=\left\{\alpha(M), 1 \otimes L^{\infty}(G)\right\}^{\prime \prime} \text {. }
$$

Proof. Since the right hand side is included in the left hand side, we want to show the converse inclusion. For this we set $y_{f, g}$ for $f, g \in K(G)$ and $y \in M$ by

$$
y_{f, 8} \equiv \int\left(1 \otimes_{t^{-1}} f\right) \alpha\left(\alpha_{t}^{-1}(y)\right) g(t) d t
$$


Since $g \in K(G)$ and $\left\|\left(1 \bigotimes_{t^{-1}} f\right) \alpha\left(\alpha_{t}^{-1}(y)\right)\right\| \leqq\|f\|_{\infty}\|y\|$, the right hand side of (8.1) is Bochner integrable and hence $y_{f, g}$ exists in $\left\{\alpha(M), 1 \otimes L^{\infty}(G)\right\}^{\prime \prime} . \quad$ If $\xi, \eta \in K(G, \mathscr{K})$, then

$$
\begin{aligned}
\left(y_{f, g} \xi \mid \eta\right) & =\iint\left(f\left(s t^{-1}\right) \alpha_{s t^{-1}}(y) g(t) \xi(s) \mid \eta(s)\right) d t d s \\
& =\iint f\left(t^{-1}\right) g(t s)\left(\alpha_{t^{-1}}(y) \xi(s) \mid \eta(s)\right) d t d s
\end{aligned}
$$

and $(s, t) \rightarrow g(t s)\left(\alpha_{t^{-1}}(y) \xi(s) \mid \eta(s)\right)$ belongs to $K(G \times G)$. If $f\left(t^{-1}\right) d t$ tends to the Dirac measure at the unit $e$ of $G$, then

$$
\left(y_{f, g} \xi \mid \eta\right) \rightarrow((y \otimes g) \xi \mid \eta) .
$$

Since $\left\|y_{f, g}\right\| \leqq\|g\|_{x}\|\Delta f\|_{1}\|y\|$ and $K(G, \mathscr{K})$ is dense in $\mathscr{K} \otimes L^{2}(G), y_{f, g}$ converges weakly to $y \otimes g$. Since $y$ and $g$ are arbitrary in $M$ and $K(G)$ respectively, $M \otimes L^{\star}(G)$ is included in $\left\{\alpha(M), 1 \otimes L^{\infty}(G)\right\}^{\prime \prime}$.

Proof of Theorem 8.1. (i) $\Rightarrow$ (ii) We set $N \equiv M \otimes_{\alpha} G$. Let $H$ be the smallest closed subgroup of $G$ satisfying $\hat{\alpha}(L) \subset N \otimes \lambda(H)^{\prime \prime}$. Since $\hat{\alpha}(y) \in N \otimes \lambda(H)^{\prime \prime}$ is equivalent to $s p_{\hat{\alpha}}(y) \subset H, H$ coincides with the closed subgroup spanned by $s p_{\hat{\alpha}}(y)$ for all $y$ in $L$. Therefore, $L$ is included in the set of all $y \in N$ with $\hat{\alpha}(y) \in N \otimes \lambda(H)^{\prime \prime}$, or in $M \otimes_{\alpha} H$ by Theorem 7.1. Let $\rho$ be the isomorphism of $M \bigotimes_{\alpha} H$ onto $M \bigotimes_{\alpha^{H}} H$ satisfying $\rho(\alpha(x))=\alpha^{H}(x)$ and $\rho(\lambda(r))=\lambda_{H}(r)$. Then

$$
\alpha^{H}(M) \subset \rho(L) \subset M \otimes_{\alpha^{H}} H .
$$

If we denote $\left(\alpha^{H}\right)^{\wedge}$ by $\beta$, then $\Gamma(\beta)=\operatorname{Ker} \alpha^{H} \mid Z(M)$ by Theorem 6.1. Since $M$ is a factor by assumption, $\Gamma(\beta)=H$.

Now, we shall show that $\rho(L) \otimes_{\beta}^{d} H$ is a factor. Since $\Gamma(\beta)=H$, we have only to prove $\beta \backslash Z(\rho(L))$ is ergodic by Theorem 6.4. For this we suppose that $\beta(z)=z \otimes 1_{H}$ for $z$ in $Z(\rho(L))$, that is, $z \in$ $Z(\rho(L))^{\beta}$. (8.2) implies that

$$
\alpha^{H}(M) \subset \rho(L)^{\beta} \subset\left(M \otimes_{\alpha^{H}} H\right)^{\beta}=\alpha^{H}(M) .
$$

Since $M$ is a factor, so is $\rho(L)^{\beta}$. Since $Z(\rho(L))^{\beta}$ is included in $Z\left(\rho(L)^{\beta}\right), z$ is a scalar operator.

On the other hand, by (8.2) we have

$$
\alpha^{H}(M) \bigotimes_{\beta}^{d} H \subset \rho(L) \bigotimes_{\beta}^{d} H \subset\left(M \bigotimes_{\alpha^{H}} H\right) \bigotimes_{\beta}^{d} H .
$$

If we apply Takesaki's duality to (8.3), we have an isomorphism $\rho^{\prime}$ of 
$\left(M \bigotimes_{\alpha^{H}} H\right) \bigotimes_{\beta}^{d} H$ onto $M \otimes B\left(L^{2}(H)\right)$ as in the proof of Theorem 2.1 such that

$$
M \otimes L^{\infty}(H) \subset \rho^{\prime}\left(\rho(L) \bigotimes_{\beta}^{d} H\right) \subset M \otimes B\left(L^{2}(H)\right),
$$

where the first inclusion is obtained by Lemma 8.2. Since $\rho(L) \bigotimes_{\beta}^{d} H$ is a factor as shown in the above, we have $\rho^{\prime}\left(\rho(L) \bigotimes_{\beta}^{d} H\right)=M \otimes B\left(L^{2}(H)\right)$ by [6, Lemma III.4.2]. Therefore

$$
\rho(L) \bigotimes_{\beta}^{d} H=\left(M \bigotimes_{\alpha^{H}} H\right) \bigotimes_{\beta}^{d} H,
$$

which implies $\rho(L)=M \bigotimes_{\alpha^{H}} H$ by (2.7) and hence $L=M \bigotimes_{\alpha} H$.

(ii) $\Rightarrow$ (i) We have only to show that $\hat{\alpha}(L) \subset L \otimes R(G)$. Since

$$
\hat{\alpha}(\alpha(x))=\alpha(x) \otimes 1_{G} \quad \text { and } \quad \hat{\alpha}\left(\lambda_{1}(r)\right)=\lambda_{1}(r) \otimes \lambda(r)
$$

and $L=M \otimes_{\alpha} H$, we have $\hat{\alpha}(L) \subset L \otimes \lambda(H)^{\prime \prime} \subset L \otimes R(G)$.

The dual version of Theorem 8.1 is the following.

THEOREM 8.3. Let $\beta$ be a dual action of $G$ on $N$ and $L$ a von Neumann subalgebra with $\beta(N) \subset L \subset N \bigotimes_{\beta}^{d} G$. If $N \bigotimes_{\beta}^{d} G$ is a factor, then the following two conditions are equivalent:

(i) $L$ is $\beta^{d}$ invariant; and

(ii) there exists a closed subgroup $H$ of $G$ such that $L=N \bigotimes_{\beta}^{d}(H \backslash G)$ (or $L=\left\{x \in N \bigotimes_{\beta}^{d} G: \hat{\beta}_{t}(x)=x, t \in H\right\}$ ).

Proof. We may assume without any loss of generality that $N$ is standard.

(i) $\Rightarrow$ (ii) Let $\beta^{\prime}$ be the dual action of $G$ on $\beta(N)^{\prime}$ defined by (1.10). Theorem 1.1 gives an action $\alpha$ of $G$ on $\left(N \bigotimes_{\beta}^{d} G\right)^{\prime}$ satisfying

$$
\left\{\beta(N)^{\prime}, \beta^{\prime}\right\} \sim\left\{\left(N \bigotimes_{\beta}^{d} G\right)^{\prime} \bigotimes_{\alpha} G, \hat{\alpha}\right\}
$$

namely, there exists an isomorphism $\rho$ of $\beta(N)^{\prime}$ onto $\left(N \bigotimes_{\beta}^{d} G\right)^{\prime} \bigotimes_{\alpha} G$ satisfying $\hat{\alpha} \circ \rho=\rho \otimes \iota \circ \beta^{\prime}$.

Since $L$ is $\beta^{d}$ invariant by (i), $\operatorname{Ad} 1 \otimes W^{*}\left(L \otimes 1_{G}\right)$ is included in $L \otimes R(G)$. Since $\left[W, 1 \otimes \lambda^{\prime}(r)\right]=0, A d 1 \otimes W\left(L^{\prime} \otimes 1_{G}\right)$ is included in $L^{\prime} \otimes R(G)$. Therefore $L^{\prime}$ is $\beta^{\prime}$ invariant. Moreover, $N \bigotimes_{\beta}^{d} G$ is a factor by assumption and

$$
\left(N \bigotimes_{\beta}^{d} G\right)^{\prime} \subset L^{\prime} \subset \beta(N)^{\prime}
$$

Therefore we can apply Theorem 8.1 to $\left\{\left(N \bigotimes_{\beta}^{d} G\right)^{\prime}, \alpha\right\}$ and obtain a 
closed subgroup $H$ of $G$ satisfying

$$
\rho\left(L^{\prime}\right)=\left(N \otimes_{\beta}^{d} G\right)^{\prime} \bigotimes_{\alpha} H .
$$

Let's recall the proof of [17, Theorem 8.1]. Then $\rho$ is of the form Ad $1 \otimes V^{\prime} \circ \beta^{\prime}$ and it satisfies

$$
\rho(y)=\alpha(y) \quad \text { and } \quad \rho\left(\lambda_{1}^{\prime}(r)\right)=1 \otimes 1_{G} \otimes \lambda(r)
$$

for $y \in\left(N \bigotimes_{\beta}^{d} G\right)^{\prime}$ and $r \in G$. Therefore $L^{\prime}$ is generated by $\left(N \bigotimes_{\beta}^{d} G\right)^{\prime}$ and $1 \otimes \lambda^{\prime}(H)$ and hence

$$
L=\left(N \bigotimes_{\beta}^{d} G\right) \cap\left(1 \otimes \lambda^{\prime}(H)\right)^{\prime}=N \bigotimes_{\beta}^{d}(H \backslash G) .
$$

(ii) $\Rightarrow$ (i) The commutant of $L \equiv N \bigotimes_{\beta}^{d}(H \backslash G)$ is generated by $\left(N \otimes_{\beta}^{d} G\right)^{\prime}$ and $1 \otimes \lambda^{\prime}(H)$. Since $\beta^{\prime}(y)=y \otimes 1_{G}$ for $y \in\left(N \bigotimes_{\beta}^{d} G\right)^{\prime}$ by Theorem 1.1 and

$$
A d 1 \otimes W\left(1 \otimes \lambda^{\prime}(r) \otimes 1_{G}\right)=1 \otimes \lambda^{\prime}(r) \otimes \lambda(r)
$$

it follows that $L^{\prime}$ is $\beta^{\prime}$ invariant. Therefore $A d 1 \otimes W\left(L^{\prime} \otimes 1_{G}\right)$ is included in $L^{\prime} \otimes R(G)$. Since $\left[W, 1 \otimes \lambda^{\prime}(r)\right]=0, L$ is $\beta^{d}$ invariant similarly as before.

TheOrem 8.4. Assume that $G$ is separable. If

(i) $N$ is a factor, and

(ii) $\beta$ is integrable and $\Gamma(\tilde{\beta})=G$,

then there exists a bijective correspondence between the closed subgroups $H$ of $G$ and the $\beta$ invariant von Neumann subalgebras $L$ of $N$ containing $N^{\beta}$ in such a way that

$$
\begin{aligned}
& L_{H}=\left\{y \in N: \beta(y) \in N \otimes \lambda(H)^{\prime \prime}\right\} \\
& H_{L}=\cap\left\{H^{\prime}: \beta(L) \subset N \otimes \lambda\left(H^{\prime}\right)^{\prime \prime}\right\},
\end{aligned}
$$

where $H^{\prime}$ runs over closed subgroups of $G$.

Proof. Let $K$ be an infinite dimensional Hilbert space with $\operatorname{dim} K \geqq$ $\operatorname{dim} L^{2}(G)$. We put $\bar{N} \equiv N \otimes B(K)$ and $\bar{\beta} \equiv \iota \otimes \sigma \circ \beta \otimes \iota$. Then $\{\bar{N}, \bar{\beta}\}$ is a covariant dual system. Since $K$ is infinite dimensional, $\bar{N}^{\bar{\beta}}=$ $N^{\beta} \otimes B(K)$ and $\operatorname{dim} K \geqq \operatorname{dim} L^{2}(G)$, it follows that $\bar{\beta}$ is of infinite multiplicity. Since $\beta$ is integrable by assumption, so is $\bar{\beta}$. If $x \in N$, $y \in B(K), z \in B\left(L^{2}(G)\right)$ and $f \in L^{\infty}(G)$, then 


$$
\begin{aligned}
& \pi \circ[\bar{\beta}](x \otimes y \otimes z)=[\beta] \otimes \iota(x \otimes z \otimes y) \\
& \pi\left(1_{N} \otimes 1_{K} \otimes 1_{G} \otimes f\right)=1_{N} \otimes 1_{G} \otimes f \otimes 1_{K}
\end{aligned}
$$

where $\pi \equiv \iota_{N} \otimes \iota \otimes \sigma \circ \iota_{N} \otimes \sigma \otimes \iota$. Therefore

$$
\pi\left(\left(\bar{N} \otimes B\left(L^{2}(G)\right) \otimes_{[\bar{\beta}]}^{d} G\right)=\left(\left(N \otimes B\left(L^{2}(G)\right) \bigotimes_{[\beta]}^{d} G\right) \otimes B(K) .\right.\right.
$$

Since $\Gamma([\beta])=\Gamma(\tilde{\beta})=G$ by (ii), it follows from Corollary 6.2 that $\Gamma(\tilde{\bar{\beta}})=\Gamma([\bar{\beta}])=G$. Since $\bar{N}^{\bar{\beta}}$ is properly infinite, Theorem 6.7 implies that $\bar{\beta}$ is dominant. Therefore $\{\bar{N}, \bar{\beta}\}$ is equivalent to $\left\{M \otimes_{\alpha} G, \hat{\alpha}\right\}$, where $M \equiv \bar{N} \bigotimes_{\bar{\beta}}^{d} G$ and $\alpha \equiv \hat{\bar{\beta}}$. Since $N$ is a factor by assumption (i), $\bar{N}$ is also a factor and hence $\bar{\beta}$ is ergodic on $Z(\bar{N})$. Since $\Gamma(\tilde{\bar{\beta}})=G, M$ is a factor by Theorem 6.4 .

Now, suppose that $L$ is $\beta$ invariant. Put $\bar{L} \equiv L \otimes B(K)$. Then $\bar{L}$ is $\bar{\beta}$ invariant. Since $\bar{N}^{\bar{\beta}} \subset \bar{L} \subset \bar{N}, \alpha(M) \subset \bar{L} \subset M \otimes_{\alpha} G$. Therefore we can apply Theorem 8.1 to $\{M, \alpha\}$ and obtain

$$
\bar{L}_{H_{\bar{L}}}=\bar{L} \quad \text { and } \quad H_{\bar{L}_{H}}=H \quad(\text { By Theorem } 7.1) .
$$

Now, since $\beta(L) \subset N \otimes \lambda(H)^{\prime \prime}$ is equivalent to $\bar{\beta}(\bar{L}) \subset \bar{N} \otimes \lambda(H)^{\prime \prime}$, we have $H_{L}=H_{\bar{L}}$. Therefore, if $x \in L_{H_{L}}$, then $x \otimes 1_{K} \in \bar{L}_{H_{\bar{L}}}=\bar{L}$ and hence $x \in L$. Since $L \subset L_{H_{L}}$ is clear, we have $L=L_{H_{L}}$.

Moreover, since $\bar{L}_{H}=L_{H} \otimes B(K)=\left(L_{H}\right)^{-}$, we have $H=H_{\bar{L}_{H}}=$ $H_{L_{H} \otimes B(K)}=H_{L_{H}}$.

9. Appendix. 1. As shown by Araki and Haagerup $[1,9]$, each action $\alpha$ of $G$ on a standard $M$ is implemented by a unitary $u$ in $B(\mathscr{H}) \otimes L^{\infty}(G)$ satisfying

$$
u \otimes 1_{G}(\iota \otimes \sigma)\left(u \otimes 1_{G}\right)=\iota \otimes \delta(u)
$$

in such a way $\alpha(x)=A d u\left(x \otimes 1_{G}\right)$ by Proposition 3.4. Then the commutant of $M \otimes_{\alpha} G$ is given by Digerness, [7, 10, 16] as follows:

$$
\left(M \otimes_{\alpha} G\right)^{\prime}=\left\{M^{\prime} \otimes 1_{G}, u\left(1 \otimes R(G)^{\prime}\right) u^{*}\right\}^{\prime \prime} .
$$

The dual version of this result is the following:

THEOREM 9.1. If a dual action $\beta$ of $G$ on a standard $N$ is implemented by a unitary $w$ in $B(\mathscr{K}) \otimes R(G)$ satisfying

$$
w^{*} \otimes 1_{G}(\iota \otimes \sigma)\left(w^{*} \otimes 1_{G}\right)=\iota \otimes \gamma\left(w^{*}\right)
$$


in such a way $\beta(y)=A d w^{*}\left(y \otimes 1_{G}\right)$, then

$$
\left(N \bigotimes_{\beta}^{d} G\right)^{\prime}=\left\{N^{\prime} \otimes 1_{G}, w^{*}\left(1 \otimes L^{\infty}(G)\right) w\right\}^{\prime \prime} .
$$

Proof. We set

$$
\pi(f) \equiv A d w^{*}(1 \otimes f)
$$

for $f \in L^{\infty}(G)$. First we shall show $\pi(f) \in\left(N \bigotimes_{\beta}^{d} G\right)^{\prime}$. For this it suffices to show that $[\pi(f), 1 \otimes g]=0$ for all $g \in L^{\infty}(G)$. It is clear that $\pi(f) \in \beta(N)^{\prime}$. Since $\iota \otimes \sigma\left(w \otimes 1_{G}\right)$ belongs to $B(\mathscr{K}) \otimes 1_{G} \otimes R(G)$ and since

$$
(1 \otimes W)\left(w^{*} \otimes 1_{G}\right) \sigma\left(w^{*} \otimes 1_{G}\right)=\left(w^{*} \otimes 1_{G}\right)(1 \otimes W)
$$

by $(9.1)$, we have

$A d 1 \otimes W \circ A d w^{*} \otimes 1_{G}\left(1 \otimes f \otimes 1_{G}\right)=A d w^{*} \otimes 1_{G}\left(1 \otimes f \otimes 1_{G}\right)$

and hence $\beta^{\prime}(\pi(f))=\pi(f) \otimes 1_{G}$, where $\beta^{\prime}$ is a dual action of $G$ on $\beta(N)^{\prime}$ defined by (1.10). Then $\pi(f) \in\left(1 \otimes L^{\infty}(G)\right)^{\prime}$ by the argument given after Theorem 7.1.

Now, as $M \equiv N \bigotimes_{\beta}^{d} G$ is globally invariant under $A d 1 \otimes \lambda^{\prime}(t)$, we can define an action of $G$ on $M^{\prime}$ by $\alpha_{t} \equiv A d 1 \otimes \lambda^{\prime}(t)\left\lceil M^{\prime}\right.$. Since $\pi$ is an isomorphism of $L^{\infty}(G)$ into $M^{\prime}$ satisfying $\alpha_{t}^{\circ} \pi=\pi \circ A d \lambda^{\prime}(t), M^{\prime}$ is generated by $\pi\left(L^{\infty}(G)\right)$ and $\left(M^{\prime}\right)^{\alpha}$ by $[16 ; 17$, Theorem 8.3]. Besides

$$
\left(M^{\prime}\right)^{\alpha}=M^{\prime} \cap\left(1 \otimes R(G)^{\prime}\right)^{\prime}=\left\{\beta(N), 1 \otimes L^{\infty}(G), 1 \otimes R(G)^{\prime}\right\}^{\prime} .
$$

It suffices to show the right hand side is $N^{\prime} \otimes 1_{G}$.

Since $\{N, \beta\}$ and $\{\beta(N), \iota \otimes \gamma\}$ are equivalent, it follows that

$$
\begin{aligned}
\beta & \otimes \iota\left(\left\{\beta(N), 1 \otimes L^{\infty}(G), 1 \otimes R(G)^{\prime}\right\}^{\prime \prime}\right) \\
& =\left\{\iota \otimes \gamma(\beta(N)), 1 \otimes 1_{G} \otimes L^{\infty}(G), 1 \otimes 1_{G} \otimes R(G)^{\prime}\right\}^{\prime \prime},
\end{aligned}
$$

which is $\beta(N) \otimes B\left(L^{2}(G)\right)$ by [16, (38) in the proof of Theorem 3]. Therefore $\left(M^{\prime}\right)^{\alpha}=N^{\prime} \otimes 1_{G}$.

Corollary 9.2. $N \otimes B\left(L^{2}(G)\right)=\left\{\beta(N), 1 \otimes B\left(L^{2}(G)\right)\right\}^{\prime \prime}$.

2. Haga's factorization holds always for a regular action. Namely, if $\alpha$ is regular, $M \otimes_{\alpha} G$ is isomorphic to $M^{\alpha} \otimes B\left(L^{2}(G)\right)$ by Theorems 2.5 and 5.6. However, as for the converse, we have only the following proposition for an abelian $G$. 
Proposition 9.3. If $G$ is abelian, the following two conditions are equivalent :

(i) $\alpha$ is regular; and

(ii) there is an isomorphism $\rho$ of $M \otimes_{\alpha} G$ onto $M^{\alpha} \otimes B\left(L^{2}(G)\right)$ such that $\rho^{-1}\left(M^{\alpha} \otimes 1_{G}\right)$ is $\hat{\alpha}$ invariant.

Proof. (i) $\Rightarrow$ (ii). If $\alpha$ is regular, $\{M, \alpha\}$ is equivalent to $\left\{M^{\alpha} \bigotimes_{\beta}^{d} G, \hat{\beta}\right\}$ for some covariant dual system $\left\{M^{\alpha}, \beta\right\}$. Therefore

$$
\left\{\overline{M^{\alpha}},[\beta]\right\} \sim\left\{\left(M^{\alpha}\right)^{\sim}, \bar{\beta}\right\} \sim\left\{M \otimes_{\alpha} G, \hat{\alpha}\right\}
$$

Since $G$ is abelian, $[\beta]\left(y \otimes 1_{G}\right)=y \otimes 1_{G} \otimes 1_{G}$ for $y \in M^{\alpha}$. Thus we have an isomorphism $\rho$ of $M \otimes_{\alpha} G$ onto $M^{\alpha} \otimes B\left(L^{2}(G)\right)$ such that $\rho^{-1}\left(M^{\alpha} \otimes 1_{G}\right)$ is $\hat{\alpha}$ invariant.

(ii) $\Rightarrow$ (i) We set

$$
\langle\beta\rangle \equiv \rho \otimes \iota \circ \hat{\alpha} \circ \rho^{-1} \text { and } \underline{\beta} \equiv A d 1 \otimes W^{*} \circ\langle\beta\rangle .
$$

Then we have

$$
\left\{M \otimes_{\alpha} G, \hat{\alpha}\right\} \sim\left\{M^{\alpha} \otimes B\left(L^{2}(G)\right),\langle\beta\rangle\right\} .
$$

Since $\langle\beta\rangle$ is a dual action of $G$ on $M^{\alpha} \otimes B\left(L^{2}(G)\right)$ and since $A d W^{*}\left(f \otimes 1_{G}\right)=f \otimes 1_{G}, \quad A d W^{*}\left(\lambda(r) \otimes 1_{G}\right)=\lambda(r) \otimes \lambda(r) \quad$ and Ad $W^{*}\left(1_{G} \otimes \lambda(r)\right)$ belongs to $L^{\infty}(G) \otimes R(G), \beta$ is an isomorphism of $M^{\alpha} \otimes B\left(L^{2}(G)\right)$ into $M^{\alpha} \otimes B\left(L^{2}(G)\right) \otimes R(G)$. However, $G$ is abelian by assumption, we have

$$
A d W^{*}\left(1_{G} \otimes \lambda(r)\right) \in 1 \otimes R(G) .
$$

Since $M^{\alpha} \otimes 1_{G}$ is $\langle\beta\rangle$ invariant by (ii), it is $\beta$ invariant by (9.3). Therefore there is an isomorphism $\beta$ of $M^{\alpha}$ into $M^{\alpha} \otimes R(G)$ such that

$$
\iota \otimes \sigma \circ \beta \otimes \iota=\underline{\beta}\left\lceil M^{\alpha} \otimes 1_{G} .\right.
$$

Now, we shall show that $\underline{\beta} \mid M^{\alpha} \otimes 1_{G}$ is a dual action of $G$ on $M^{\alpha} \otimes 1_{G} . \quad$ If $y \in N$, then

$$
\begin{aligned}
\underline{\beta} & \otimes \iota \circ \underline{\beta}\left(y \otimes 1_{G}\right)=\underline{\beta} \otimes \iota \circ A d 1 \otimes W^{*} \circ\langle\beta\rangle\left(y \otimes 1_{G}\right) \\
& =\iota \otimes \sigma \otimes \iota \circ \beta \otimes \iota \otimes \iota \circ A d 1 \otimes W^{*} \circ\langle\beta\rangle\left(y \otimes 1_{G}\right) \quad \text { (By (9.4)) } \\
& =\iota \otimes \sigma \otimes \iota \circ A d 1 \otimes 1_{G} \otimes W^{*} \circ \beta \otimes \iota \otimes \iota \circ\langle\beta\rangle\left(y \otimes 1_{G}\right)
\end{aligned}
$$




$$
\begin{aligned}
= & \tau \circ\langle\beta\rangle \otimes \iota \circ\langle\beta\rangle\left(y \otimes 1_{G}\right) \quad(\text { By }(9.4)) \\
& \left(\tau \equiv \iota \otimes \sigma \otimes \iota \circ A d 1 \otimes 1_{G} \otimes W^{*} \circ \iota \otimes \sigma \otimes \iota \circ A d 1 \otimes W^{*} \otimes 1_{G}\right) \\
= & \tau \circ A d 1 \otimes 1_{G} \otimes W^{*} \circ\langle\beta\rangle \otimes \iota\left(y \otimes 1_{G} \otimes 1_{G}\right) \\
= & \tau \circ A d\left(\left(1 \otimes 1_{G} \otimes W^{*}\right)\left(1 \otimes W \otimes 1_{G}\right)\right) \beta \otimes \iota\left(y \otimes 1_{G} \otimes 1_{G}\right) \\
= & A d 1 \otimes 1_{G} \otimes W^{*} \circ \underline{\beta} \otimes \iota\left(y \otimes 1_{G} \otimes 1_{G}\right),
\end{aligned}
$$

where the last equality follows from

$$
W \otimes 1_{G}(\sigma \otimes \iota) 1_{G} \otimes W=A d 1_{G} \otimes W^{*}\left(W \otimes 1_{G}\right) .
$$

Consequently, since $\beta$ is a dual action of $G$ on $M^{\alpha} \otimes 1_{G}$ and $\beta \otimes \iota=\iota \otimes \sigma \circ \beta \backslash M_{\alpha} \otimes 1_{G}, \beta$ is a dual action of $G$ on $M^{\alpha}$ and $\langle\beta\rangle=\operatorname{Ad} 1 \otimes W^{-} \circ \bar{\beta}=[\beta]$, which implies that

$$
M \sim \alpha(M)=\left(M \bigotimes_{\alpha} G\right)^{\hat{\alpha}} \sim\left(M^{\alpha} \otimes B\left(L^{2}(G)\right)\right)^{[\beta]}=M^{\alpha} \bigotimes_{\beta}^{d} G
$$

by (9.2). Therefore $\alpha$ is regular.

\section{REFERENCES}

1. H. Araki, Some properties of modular conjugation operator of von Neumann algebras and a noncommutative Radon-Nikodym theorem with a chain rule, Pacific J. Math., 50 (1974), 309-354.

2. - Structure of some von Neumann algebras with isolated discrete modular spectrum, Publ. RIMS, Kyoto Univ., 9 (1973), 1-14.

3. W. B. Arveson, On groups of automorphisms of operator algebras, J. Functional Analysis, 15 (1974), 217-243.

4. M. Choda, Normal expectations and crossed products of von Neumann algebras, Proc. Japan Acad., 50 (1974), 738-742.

5. A. Connes, Une classification des facteurs de type III, Ann. Sci. École Norm. Sup., 6 (1973), $133-252$.

6. A. Connes and M. Takesaki, The flow of weights on factors of type III, Tôhoku Math. J., to appear.

7. T. Digerness, Dual weight and the commutation theorem for crossed products of $W^{*}$-algebras, Preprint, CNRS, Marseille, 1974.

8. P. Eymard, L'algèbre de Fourier d'un groupe localement compact, Bull. Soc. Math. France, 92 (1964), 181-236.

9. U. Haagerup, The standard form of von Neumann algebras, Math. Scand., 37 (1975), 271-283.

10. - On the dual weights for crossed products of von Neumann algebras I, II, Preprints, Odense Univ., 1975.

11. — Operator valued weights in von Neumann algebras, Preprint, Odense Univ., 1975.

12. Y. Haga, Crossed products of von Neumann algebras by compact groups, Tôhoku Math. J., 28 (1976), 511-522.

13. Y. Haga and Z. Takeda, Correspondence between subgroups and subalgebras in a cross product von Neumann algebra, Tôhoku Math. J., 24 (1972), 167-190. 
14. A. Ikunishi and Y. Nakagami, On invariants $G(\sigma)$ and $\Gamma(\sigma)$ for an automorphism group of a von Neumann algebra, Publ. RIMS, Kyoto Univ., 12 (1976), 1-30.

15. M. B. Landstad, Duality theory for covariant systems, Preprint, Univ. of Trondheim, 1974.

16. - Duality for dual covariance algebras, Commun. Math. Phys., 52 (1977), 191-202.

17. Y. Nakagami, Dual action on a von Neumann algebra and Takesaki's duality for a locally compact group, Publ. RIMS, Kyoto Univ. 12 (1977), 727-775.

18. M. Nakamura and Z. Takeda, On some elementary properties of the crossed product of von Neumann algebras, Proc. Japan Acad., 34 (1958), 489-494.

19. — A Galois theory for finite factors, Proc. Japan Acad., 36 (1960), 258-260.

20. J. E. Roberts, Cross products of von Neumann algebras by group duals, Inst. Nationale di Alta Mat. Symposia Math., 20 (1976).

21. S. Strătilă, D. Voiculescu and L. Zsidó, On crossed products, Rev. Roumaine Math., 21 (1976), 1411-1449; 22 (1977), 83-117.

22. M. Takesaki, Covariance representations of $C^{*}$-algebras and their locally compact automorphism groups, Acta Math., 119 (1967), 273-303.

23. Duality for crossed products and the structure of von Neumann algebras of type III, Acta Math., 131 (1971), 249-310.

24. M. Takesaki and N. Tatsuuma, Duality and subgroups, Ann. of Math., 93 (1971), 344-364.

25. J. Tomiyama, Tensor products and projections of norm one in von Neumann algebras, Lecture Note at Univ. of Copenhagen. 1970.

Received November 12, 1976 and in revised form March 17, 1977. This work is supported in part by NSF grant No. MCS 76-06899.

UniVERSity of CALIFornia, Los ANGEles

Los ANGELEs, CA 90024

Current address: YoKoHAMA City UNIVERSITY

4646 MutsuUra-CHo, Kanazawa-Ku

YOKOHAMA, 236 JAPAN 



\section{PACIFIC JOURNAL OF MATHEMATICS \\ EDITORS}

RICHARD ARENS (Managing Editor)

University of California

Los Angeles, CA 90024

\section{R. A. Beaumont \\ University of Washington \\ Seattle, WA 98105}

C. C. MOORE

University of California

Berkeley, CA 94720

\section{J. DUGUNDJI}

Department of Mathematics

University of Southern California

Los Angeles, CA 90007

R. FINN AND J. MILGRAM

Stanford University

Stanford, CA 94305

\section{ASSOCIATE EDITORS}

\section{E. F. BECKENBACH}

B. H. NeUmanN

F. WOLF

K. YoSHIDA

\section{SUPPORTING INSTITUTIONS}

\author{
UNIVERSITY OF BRITISH COLUMBIA \\ CALIFORNIA INSTITUTE OF TECHNOLOGY \\ UNIVERSITY OF CALIFORNIA \\ MONTANA STATE UNIVERSITY \\ UNIVERSITY OF NEVADA \\ NEW MEXICO STATE UNIVERSITY \\ OREGON STATE UNIVERSITY \\ UNIVERSITY OF OREGON \\ OSAKA UNIVERSITY
}

\author{
UNIVERSITY OF SOUTHERN CALIFORNIA \\ STANFORD UNIVERSITY \\ UNIVERSITY OF HAWAII \\ UNIVERSITY OF TOKYO \\ UNIVERSITY OF UTAH \\ WASHINGTON STATE UNIVERSITY \\ UNIVERSITY OF WASHINGTON \\ AMERICAN MATHEMATICAL SOCIETY
}

The Supporting Institutions listed above contribute to the cost of publication of this Journal, but they are not owners or publishers and have no responsibility for its contents or policies.

Mathematical papers intended for publication in the Pacific Journal of Mathematics should be in typed form or offset-reproduced (not dittoed), double spaced with large margins. Underline Greek letters in red, German in green, and script in blue. The first paragraph or two must be capable of being used separately as a synopsis of the entire paper. Items of the bibliography should not be cited there unless absolutely necessary, in which case they must be identified by author and Journal, rather than by item number. Manuscripts, in duplicate, may be sent to any one of the four editors. Please classify according to the scheme of Math. Reviews, Index to Vol. 39. All other communications should be addressed to the managing editor, or Elaine Barth, University of California, Los Angeles, California, 90024.

100 reprints are provided free for each article, only if page charges have been substantially paid. Additional copies may be obtained at cost in multiples of 50 .

The Pacific Journal of Mathematics is issued monthly as of January 1966. Regular subscription rate: $\$ 72.00$ a year (6 Vols., 12 issues). Special rate: $\$ 36.00$ a year to individual members of supporting institutions.

Subscriptions, orders for back numbers, and changes of address should be sent to Pacific Journal of Mathematics, 103 Highland Boulevard, Berkeley, California, 94708.

PUBLISHED BY PACIFIC JOURNAL OF MATHEMATICS, A NON-PROFIT CORPORATION

Printed at Jerusalem Academic Press, POB 2390, Jerusalem, Israel.

Copyright (C) 1977 Pacific Journal of Mathematics All Rights Reserved 


\section{Pacific Journal of Mathematics \\ Vol. 70, No. $2 \quad$ October, 1977}

B. Arazi, A generalization of the Chinese remainder theorem ........... 289

Thomas E. Armstrong, Polyhedrality of infinite dimensional cubes .... . . . 297

Yoav Benyamini, Mary Ellen Rudin and Michael L. Wage, Continuous

images of weakly compact subsets of Banach spaces ............ 309

John Thomas Burns, Curvature functions on Lorentz 2-manifolds ......... 325

Dennis F. De Riggi and Nelson Groh Markley, Shear distality and equicontinuity .................................. 337

Claes Fernström, Rational approximation and the growth of analytic

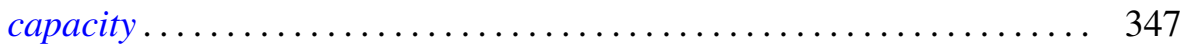

Pál Fischer, On some new generalizations of Shannon's inequality....... 351

Che-Kao Fong, Quasi-affine transforms of subnormal operators ......... 361

Stanley P. Gudder and W. Scruggs, Unbounded representations of

*-algebras........................................ 369

Chen F. King, A note on Drazin inverses .................... 383

Ronald Fred Levy, Countable spaces without points of first countability . . . 391

Eva Lowen-Colebunders, Completeness properties for convergence

spaces ......................................... 401

Calvin Cooper Moore, Square integrable primary representations ....... 413

Stanisław G. Mrówka and Jung-Hsien Tsai, On preservation of

E-compactness ................................ 429

Yoshiomi Nakagami, Essential spectrum $\Gamma(\beta)$ of a dual action on a von

Neumann algebra ................................ 437

L. Alayne Parson, Normal congruence subgroups of the Hecke groups

$G\left(2^{(1 / 2)}\right)$ and $G\left(3^{(1 / 2)}\right)$...

Louis Jackson Ratliff, Jr., On the prime divisors of zero in form rings . . . . 489

Caroline Series, Ergodic actions of product groups .................. 519

Robert O. Stanton, Infinite decomposition bases..................... 549

David A. Stegenga, Sums of invariant subspaces .................. 567 